\title{
Introduction to $L_{p}$ Sobolev Spaces via Muramatu's Integral Formula
}

\author{
Yoichi Miyazaki
}

\begin{abstract}
Muramatu's integral formula is a very useful tool for the study of Sobolev spaces, although this does not seem to be widely recognized. Most theorems in Sobolev spaces can be proved by this formula combined with basic inequalities in analysis, and it is possible to directly treat not only the whole space but also a special Lipschitz domain. In this paper, we present an introduction to $L_{p}$-based Sobolev spaces of integer order by making Muramatu's integral formula play a central role, as Cauchy's integral formula does in complex analysis. The topics we take up are approximation by smooth functions, the interpolation inequality, the Sobolev embedding theorems, the trace theorem, construction of an extension operator, complex interpolation of Sobolev spaces and real interpolation of Sobolev spaces.
\end{abstract}

Mathematics Subject Classification (2010). Primary 46E35; Secondary 46B70.

Keywords. Sobolev spac, Besov space, Hölder space, Sobolev embedding theorem, Sobolev trace theorem, complex interpolation, real interpolation.

\section{Introduction}

There are many books that cover Sobolev spaces (see $[1,7,17,18,30,32,40]$ for the books whose main themes are Sobolev spaces, and [6, 9, 10, 11, 13, 14, 16, 31, 33, 34] for the books which are related to partial differential equations), and the standard way seems to have been established. Nevertheless, we wish to propose another way of using Muramatu's integral formula [22, Corollary 1, p341] to study Sobolev spaces, especially as an introductory course of $L_{p}$-based Sobolev spaces. We collect features of Muramatu's integral formula.

- It is simple and easy to use.

- Most theorems can be proved by it with the help of basic inequalities such as Hölder's inequality, Minkowski's inequality for integrals, Young's inequality and the lemma on boundedness of integral operators. This means that we can make Muramatu's integral formula play a central role in the theory of Sobolev spaces, as Cauchy's integral formula does in complex analysis. 
- If you understand the proof of a theorem, then you can think of the proof of another theorem by yourself. This is in contrast with the standard way that requires different techniques to prove different theorems.

- It enables us to prove some theorems in one shot. In other words, we do not have to use induction on the regularity order of Sobolev spaces.

- It enables us to treat directly a special Lipschitz domain as if the domain were the whole space $\mathbb{R}^{d}$. It works for $L_{p}$-based Sobolev spaces on a special Lipschitz domain as well as the Fourier transform does for $L_{2}$-based Sobolev spaces on $\mathbb{R}^{d}$ (see $\left.[12,15,33]\right)$.

- It works well also for Sobolev spaces of negative order.

In order to demonstrate how useful Muramatu's integral formula is, we give here a short proof of the embedding theorem $W_{p}^{m}\left(\mathbb{R}^{d}\right) \subset L_{q}\left(\mathbb{R}^{d}\right)$ for $1 \leq p<q<\infty$ and $m-d / p>-d / q$ with a positive integer $m$. Let $f \in W_{p}^{m}\left(\mathbb{R}^{d}\right)$. Then Muramatu's formula or its primitive form is given by

$$
f(x)=\int_{0}^{1} \sum_{|\alpha|=m}\left[\int_{\mathbb{R}^{d}} t^{-d}\left(\partial^{\alpha} K_{\alpha}\right)\left(\frac{x-y}{t}\right) f(y) d y\right] \frac{d t}{t}+\varphi * f(x)
$$

with suitable functions $\varphi \in C_{0}^{\infty}\left(\mathbb{R}^{d}\right)$ and $K_{\alpha} \in C_{0}^{\infty}\left(\mathbb{R}^{d}\right)$ for every multi-index $\alpha$, where $\varphi * f$ stands for the convolution of $\varphi$ and $f$. Noting

$$
\left(\partial^{\alpha} K_{\alpha}\right)\left(\frac{x-y}{t}\right)=t^{|\alpha|}\left(-\frac{\partial}{\partial y}\right)^{\alpha}\left\{K_{\alpha}\left(\frac{x-y}{t}\right)\right\}
$$

and taking into account the definition of weak derivatives (or integrating by parts if $\left.f \in W_{p}^{m}\left(\mathbb{R}^{d}\right) \cap C^{\infty}\left(\mathbb{R}^{d}\right)\right)$, we can move $\partial^{\alpha}$ from $K_{\alpha}$ to $f$ to get

$$
\int_{\mathbb{R}^{d}} t^{-d}\left(\partial^{\alpha} K_{\alpha}\right)\left(\frac{x-y}{t}\right) f(y) d y=\int_{\mathbb{R}^{d}} t^{m-d} K_{\alpha}\left(\frac{x-y}{t}\right) \partial^{\alpha} f(y) d y
$$

for $|\alpha|=m$. Taking $r \in(1, \infty)$ so that $p^{-1}+r^{-1}=1+q^{-1}$, and using Young's inequality, Minkowski's inequality and the relations

$$
\left\|t^{-d} K_{\alpha}\left(\frac{\cdot}{t}\right)\right\|_{L_{r}}=t^{d\left(-1+r^{-1}\right)}\left\|K_{\alpha}\right\|_{L_{r}}=t^{d\left(-p^{-1}+q^{-1}\right)}\left\|K_{\alpha}\right\|_{L_{r}},
$$

we have

$$
\begin{aligned}
\|f\|_{L_{q}} & \leq \int_{0}^{1} \sum_{|\alpha|=m} t^{m-d}\left\|K_{\alpha}\left(\frac{\cdot}{\bar{t}}\right)\right\|_{L_{r}}\left\|\partial^{\alpha} f\right\|_{L_{p}} \frac{d t}{t}+\|\varphi\|_{L_{r}}\|f\|_{L_{p}} \\
& =\sum_{|\alpha|=m}\left\|K_{\alpha}\right\|_{L_{r}}\left\|\partial^{\alpha} f\right\|_{L_{p}} \int_{0}^{1} t^{m-d / p+d / q} \frac{d t}{t}+\|\varphi\|_{L_{r}}\|f\|_{L_{p}} \\
& \leq C\|f\|_{W_{p}^{m}}
\end{aligned}
$$

where the last integral in $t$ converges since $m-d / p+d / q>0$ by assumption. Thus we have obtained $f \in L_{q}\left(\mathbb{R}^{d}\right)$. The differentiability of $f$ is translated into the integrability with respect to the measure $t^{-1} d t$ of the function related to regularizations of $f$. This is the essence of Muramatu's method. 
Muramatu [22] devised not only formula (1.1) but also the second integral formula [22, Theorem 1, p344], which is obtained by a repeated use of (1.1), and which is more complicated than (1.1). Combining the second formula with the LittlewoodPaley theory of $L_{p}$ boundedness of operators acting on Hilbert-space-valued functions, he investigated Sobolev spaces $W_{p}^{\sigma}(\Omega)$ as well as Besov spaces $B_{p q}^{\sigma}(\Omega)$ for all $\sigma \in \mathbb{R}$. As for Sobolev spaces, he showed that a function $f(x)$ belongs to $W_{p}^{\sigma}(\Omega)$ if and only if its regularization $u(t, x)=\int_{\mathbb{R}^{d}} t^{-d} K((x-y) / t) f(y) d y$ with a suitable $C^{\infty}$ function $K$ satisfies $t^{-\sigma} u(t, x) \in L_{p}\left(\Omega, L_{2}\left(I, t^{-1} d t\right)\right)$ with $I=(0,1)$, and proved the theorems on Sobolev spaces by reducing the matters to the properties of $L_{p}\left(\Omega, L_{2}\left(I, t^{-1} d t\right)\right)$.

In this paper, we give an introduction to Sobolev spaces $W_{p}^{m}(\Omega)$ with integer $m$ and $1 \leq p<\infty$ on a domain $\Omega$ of $\mathbb{R}^{d}$, making full use of Muramatu's integral formula. Our viewpoint in this paper is different from that of Muramatu [22] who aimed at full generalities. We put emphasis on simplicity and try to make the presentation as accessible as possible. Since formula (1.1) is powerful enough for Sobolev spaces of integer order, we can prove the theorems without using the second integral formula and the characterization of Sobolev spaces by $L_{p}\left(\Omega, L_{2}\left(I, t^{-1} d t\right)\right)$, which are required for Sobolev spaces of fractional order. The domain we treat in this paper is the whole space $\mathbb{R}^{d}$ or a special Lipschitz domain including the half space. As for the treatment of bounded Lipschitz domains we will make some remarks in Section 11.

This paper is organized as follows. In Section 2 we derive Muramatu's integral formula and apply it to the interpolation inequality. Sections 3 and 4 are devoted to the Sobolev embedding theorems into another Sobolev space and the HölderZygmund space, respectively. In Section 5 we prove the trace theorem for the half space after defining the Besov space which is a suitable function space for describing the boundary values. In Section 6 we present the convergence theorem which enables us to investigate Sobolev spaces further. The proof for $p=2$ is given, and the proof for $p \neq 2$ is postponed to the Appendix. As an application we show the interpolation inequality concerning pure derivatives. In Section 7 we construct an extension operator from $W_{p}^{m}(\Omega)$ to $W_{p}^{m}\left(\mathbb{R}^{d}\right)$. Sections 8 and 9 are devoted to complex and real interpolations of Sobolev spaces, respectively. In Section 10 we define Sobolev spaces of negative order, and show that Muramatu's formula also works well for the case of negative order. As an application we give a short proof of a lemma which leads to the regularity theorem for elliptic equations in divergence form. In Section 11 we give a survey of the related literature and comparisons with the methods presented there. In the Appendix we complete the proof of the convergence theorem.

The interdependence of the sections is indicated in Figure 1. This paper is self-contained except for the proof of Theorem 3.1 in a certain critical case.

\section{Muramatu's integral formula}

Let $1 \leq p \leq \infty$ and $m \in \mathbb{N}=\{1,2,3, \ldots\}$. The Sobolev space $W_{p}^{m}(\Omega)$ on a domain $\Omega$ of $\mathbb{R}^{d}$ is the collection of functions $f \in L_{p}(\Omega)$ satisfying $\partial^{\alpha} f \in L_{p}(\Omega)$ for all 


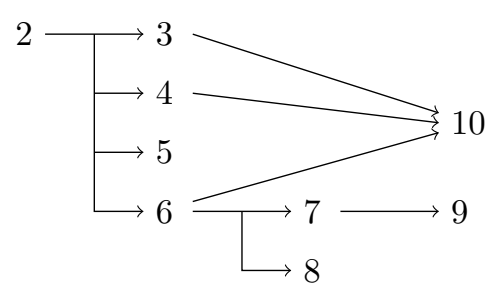

FIGURE 1

multi-indices $\alpha=\left(\alpha_{1}, \ldots, \alpha_{d}\right) \in \mathbb{N}_{0}^{d}$ with $|\alpha| \leq m$. Here $\mathbb{N}_{0}=\mathbb{N} \cup\{0\}$ and

$$
\partial^{\alpha}=\partial_{x}^{\alpha}=\partial_{1}^{\alpha_{1}} \cdots \partial_{d}^{\alpha_{d}}, \quad \partial_{j}=\partial_{x_{j}}=\frac{\partial}{\partial x_{j}} \quad(j=1, \ldots, d)
$$

for $x=\left(x_{1}, \ldots, x_{d}\right) \in \mathbb{R}^{d}$. The derivative $\partial^{\alpha} f$ should be interpreted as the weak derivative or in the distributional sense, so $\partial^{\alpha} f$ is observed via the identity

$$
\int_{\Omega} \partial^{\alpha} f(x) \phi(x) d x=(-1)^{|\alpha|} \int_{\Omega} f(x) \partial^{\alpha} \phi(x) d x, \quad \text { for all } \phi \in C_{0}^{\infty}(\Omega) .
$$

We often write $f^{(\alpha)}$ for $\partial^{\alpha} f$. The Sobolev space $W_{p}^{m}(\Omega)$ is a Banach space, being equipped with the norm

$$
\|f\|_{W_{p}^{m}(\Omega)}=\sum_{|\alpha| \leq m}\left\|\partial^{\alpha} f\right\|_{L_{p}(\Omega)} .
$$

This fact follows by the completeness of $L_{p}(\Omega)$ and the following lemma.

Lemma 2.1. Let $1 \leq p \leq \infty$. Let $\left\{f_{n}\right\}_{n=1}^{\infty}$ be a sequence in $L_{p}(\Omega)$ such that for a fixed multi-index $\alpha$, the weak derivative $\partial^{\alpha} f_{n}$ belongs to $L_{p}(\Omega)$ and that $\partial^{\alpha} f_{n}$ converges in $L_{p}(\Omega)$. We denote by $f$ the limit of $f_{n}$ in $L_{p}(\Omega)$. Then $f$ has the weak derivative $\partial^{\alpha} f$ which belongs to $L_{p}(\Omega)$, and satisfies

$$
\partial^{\alpha} f=\partial^{\alpha}\left(\lim _{n \rightarrow \infty} f_{n}\right)=\lim _{n \rightarrow \infty} \partial^{\alpha} f_{n}
$$

Proof. It follows that

$$
\int_{\Omega} \partial^{\alpha} f_{n}(x) \phi(x) d x=(-1)^{|\alpha|} \int_{\Omega} f_{n}(x) \partial^{\alpha} \phi(x) d x, \quad \text { for all } \phi \in C_{0}^{\infty}(\Omega) .
$$

Writing $f_{\alpha}$ for the limit of $\partial^{\alpha} f_{n}$ in $L_{p}(\Omega)$, and letting $n \rightarrow \infty$, we get

$$
\int_{\Omega} f_{\alpha}(x) \phi(x) d x=(-1)^{|\alpha|} \int_{\Omega} f(x) \partial^{\alpha} \phi(x) d x .
$$

By the definition of weak derivatives we know that $\partial^{\alpha} f=f_{\alpha} \in L_{p}(\Omega)$.

Definition 2.2. A domain $\Omega$ of $\mathbb{R}^{d}$ is said to be a special Lipschitz domain if there is a Lipschitz continuous function $\kappa: \mathbb{R}^{d-1} \rightarrow \mathbb{R}$ such that $\Omega$ is written as

$$
\Omega=\left\{x=\left(x^{\prime}, x_{d}\right): x_{d}>\kappa\left(x^{\prime}\right)\right\}
$$

after a suitable rotation. Here we mean by Lipschitz continuity that there exists $M>0$ such that $\left|\kappa\left(x^{\prime}\right)-\kappa\left(y^{\prime}\right)\right| \leq M\left|x^{\prime}-y^{\prime}\right|$ for all $x^{\prime}, y^{\prime} \in \mathbb{R}^{d-1}$. 
Throughout this paper, if not specified, we assume that

$\Omega$ is the whole space $\mathbb{R}^{d}$ or a special Lipschitz domain.

Under assumption (2.1) there exists an (infinite) open cone $\Gamma$ such that

$$
x+\Gamma \subset \Omega \text { for all } x \in \Omega .
$$

Here an open cone is a subset of $\mathbb{R}^{d}$ which is written in the form

$$
\left\{x \in \mathbb{R}^{d}: x \cdot \nu /|x|>\cos \theta\right\}
$$

with a unit vector $\nu \in \mathbb{R}^{d}$ and an angle $\theta \in(0, \pi / 2]$.

Let us derive Muramatu's integral formula, assuming $1 \leq p<\infty$. Let $\Gamma$ be the cone satisfying (2.2), and set

$$
\Gamma_{0}=\{x \in \Gamma:|x|<1\} .
$$

Inclusion (2.2) implies $x+t \Gamma_{0} \subset \Omega$ for all $x \in \Omega$ and $t>0$. For a function $K$ on $\mathbb{R}^{d}$ and $t>0$ we define the rescaling $K_{t}$ by

$$
K_{t}(x)=t^{-d} K\left(\frac{x}{t}\right) .
$$

When we consider a function $K_{\alpha}$, which depends on a multi-index $\alpha$, we write its rescaling as $\left(K_{\alpha}\right)_{t}$, i.e. $\left(K_{\alpha}\right)_{t}(x)=t^{-d} K_{\alpha}(x / t)$.

Take a function $\varphi \in C_{0}^{\infty}\left(\mathbb{R}^{d}\right)$ satisfying

$$
\int_{\mathbb{R}^{d}} \varphi(x) d x=1, \quad \operatorname{supp} \varphi \subset-\Gamma_{0} .
$$

For $f \in L_{p}(\Omega)$ we consider the convolution of the rescaling $\varphi_{t}$ and $f$ :

$$
\varphi_{t} * f(x)=\int_{\Omega} \varphi_{t}(x-y) f(y) d y=\int_{\Omega} t^{-d} \varphi\left(\frac{x-y}{t}\right) f(y) d y
$$

for $x \in \Omega$. Clearly, $\varphi_{t} * f(x) \in C^{\infty}((0, \infty) \times \Omega)$ as a function of $t$ and $x$, and

$$
\partial_{t}^{k} \partial_{x}^{\alpha}\left\{\varphi_{t} * f(x)\right\}=\int_{\Omega} \partial_{t}^{k} \partial_{x}^{\alpha}\left\{\varphi_{t}(x-y)\right\} f(y) d y .
$$

If we set

$$
K(x)=\sum_{j=1}^{d} \partial_{j}\left\{x_{j} \varphi(x)\right\}
$$

then

$$
\frac{\partial}{\partial t}\left\{\varphi_{t}(x)\right\}=-t^{-1} K_{t}(x)
$$

holds, since both sides are equal to $-t^{-d-1}\left\{d \varphi(x / t)+\sum_{j=1}^{d}\left(x_{j} / t\right) \partial_{j} \varphi(x / t)\right\}$. Integrating $\partial_{t}\left\{\varphi_{t} * f(x)\right\}$ over the interval $[\epsilon, \lambda]$ with $0<\epsilon<\lambda$, we have

$$
\varphi_{\epsilon} * f(x)=\int_{\epsilon}^{\lambda} K_{t} * f(x) \frac{d t}{t}+\varphi_{\lambda} * f(x) .
$$

Let $E_{0} f$ denote the extension of $f$ to $\mathbb{R}^{d}$ by zero, namely

$$
E_{0} f(x)=f(x), \quad \text { for } x \in \Omega ; \quad E_{0} f(x)=0, \quad \text { for } x \in \mathbb{R}^{d} \backslash \Omega .
$$


Then $\varphi_{\epsilon} * f$ is considered as the restriction to $\Omega$ of the function $\varphi_{\epsilon} * E_{0} f$ in $\mathbb{R}^{d}$. By Young's inequality

$$
\left\|\varphi_{\epsilon} * f\right\|_{L_{p}(\Omega)} \leq\left\|\varphi_{\epsilon} * E_{0} f\right\|_{L_{p}\left(\mathbb{R}^{d}\right)} \leq\|\varphi\|_{L_{1}\left(\mathbb{R}^{d}\right)}\|f\|_{L_{p}(\Omega)} .
$$

Since $\varphi_{\epsilon} * E_{0} f \rightarrow E_{0} f$ in $L_{p}\left(\mathbb{R}^{d}\right)$ as $\epsilon \rightarrow 0$, we have

$$
\varphi_{\epsilon} * f \rightarrow f, \quad \text { in } L_{p}(\Omega) .
$$

Letting $\epsilon \rightarrow 0$ in (2.8), we get

$$
f=\int_{0}^{\lambda} K_{t} * f \frac{d t}{t}+\varphi_{\lambda} * f, \quad \text { for } f \in L_{p}(\Omega)
$$

where the integral $\int_{0}^{\lambda}$ should be interpreted as $\lim _{\epsilon \rightarrow 0} \int_{\epsilon}^{\lambda}$ in the norm of $L_{p}(\Omega)$.

It is essential that $K$ in (2.11) consists of the derivatives of some functions, namely $K=\sum_{j=1}^{d} \partial_{j} K_{j}$ with $K_{j}(x)=x_{j} \varphi(x)$ by (2.6), and that the support of $K_{j}$ is also contained in $-\Gamma_{0}$. The advantage of supp $K_{j} \subset-\Gamma_{0}$ is that if $x \in \Omega$ then the function $\left(K_{j}\right)_{t}(x-\cdot)$ is supported in $x+t \Gamma_{0} \subset \Omega$ and hence that we can handle distributional derivatives of $f$ in $\Omega$. Indeed, if $f \in W_{p}^{1}(\Omega)$ then we have, for $x \in \Omega$,

$$
\left(\partial_{j} K_{j}\right)_{t} * f(x)=-t \int_{\Omega} \partial_{y_{j}}\left\{\left(K_{j}\right)_{t}(x-y)\right\} f(y) d y=t\left(K_{j}\right)_{t} * \partial_{j} f(x) .
$$

This combined with (2.11) yields

$$
f=\int_{0}^{\lambda} \sum_{j=1}^{d} t\left(K_{j}\right)_{t} * \partial_{j} f \frac{d t}{t}+\varphi_{\lambda} * f, \quad \text { for } f \in W_{p}^{1}(\Omega) .
$$

Thus by moving the derivatives from $K$ to $f$ we have succeeded in producing a factor $t$ in the integrand. If we could move the derivatives $k$ times from $K$ to $f$, then we could produce a factor $t^{k}$, which makes the integrand more integrable in $t$. Muramatu's ingenuity lies in his construction of $\varphi$ which enables us to express $K$ as the sum of higher-order derivatives of some functions.

For $x=\left(x_{1}, \ldots, x_{d}\right) \in \mathbb{R}^{d}$ and a multi-index $\alpha=\left(\alpha_{1}, \ldots, \alpha_{d}\right)$ we use the symbol

$$
x^{\alpha}=x_{1}^{\alpha_{1}} \cdots x_{d}^{\alpha_{d}} .
$$

Lemma 2.3. Let $N \in \mathbb{N}$, and $\omega \in C_{0}^{\infty}\left(\mathbb{R}^{d}\right)$. If we set

$$
\varphi(x)=\sum_{|\alpha|<N} \frac{1}{\alpha !} \partial^{\alpha}\left(x^{\alpha} \omega(x)\right), \quad K(x)=\sum_{|\alpha|=N} \frac{N}{\alpha !} \partial^{\alpha}\left(x^{\alpha} \omega(x)\right),
$$

then (2.7) holds for $x \in \mathbb{R}^{d}$ and $t>0$.

Proof. By the calculation that derived (2.7) from (2.6) it suffices to show that relation (2.6) holds if $\varphi$ and $K$ are defined by (2.13). Let $e_{j}$ be the multi-index of length 1 whose $j$ th component is 1 . Using

$$
\partial^{\alpha}\left(x^{\alpha+e_{j}} \omega(x)\right)=x_{j} \partial^{\alpha}\left(x^{\alpha} \omega(x)\right)+\alpha_{j} \partial^{\alpha-e_{j}}\left(x^{\alpha} \omega(x)\right),
$$


which follows by the Leibniz formula, we have

$$
\begin{aligned}
\sum_{j=1}^{d} \partial_{j}\left(x_{j} \varphi(x)\right) & =\sum_{j=1}^{d} \sum_{|\alpha|<N} \frac{1}{\alpha !} \partial_{j}\left\{x_{j} \partial^{\alpha}\left(x^{\alpha} \omega(x)\right)\right\} \\
& =\sum_{j=1}^{d} \sum_{|\alpha|<N}\left\{\frac{\alpha_{j}+1}{\left(\alpha+e_{j}\right) !} \partial^{\alpha+e_{j}}\left(x^{\alpha+e_{j}} \omega(x)\right)-\frac{\alpha_{j}}{\alpha !} \partial^{\alpha}\left(x^{\alpha} \omega(x)\right)\right\} .
\end{aligned}
$$

Let $|\beta|>0$ with $\beta=\left(\beta_{1}, \ldots, \beta_{d}\right)$. If $\beta_{j} \geq 1$ then there is a unique multi-index $\alpha^{j}=\left(\alpha_{1}^{j}, \ldots, \alpha_{d}^{j}\right)$ satisfying $\beta=\alpha^{j}+e_{j}$. So $|\beta|=\sum_{j: \beta_{j} \geq 1} \beta_{j}=\sum_{j: \beta_{j} \geq 1}\left(\alpha_{j}^{j}+1\right)$. Therefore,

$$
\sum_{j=1}^{d} \partial_{j}\left(x_{j} \varphi(x)\right)=\sum_{0<|\beta| \leq N} \frac{|\beta|}{\beta !} \partial^{\beta}\left(x^{\beta} \omega(x)\right)-\sum_{|\alpha|<N} \frac{|\alpha|}{\alpha !} \partial^{\alpha}\left(x^{\alpha} \omega(x)\right),
$$

which gives (2.6).

Definition 2.4. For $N \in \mathbb{N}_{0}$ we denote by $\mathcal{K}_{\Gamma}^{N}$ the set of functions $K$ written in the form

$$
K=\sum_{|\alpha|=N} \partial^{\alpha} K_{\alpha}
$$

with some $K_{\alpha} \in C_{0}^{\infty}\left(\mathbb{R}^{d}\right)$ supported in $-\Gamma_{0}$. (Recall that $\Gamma_{0}$ is defined from $\Gamma$ by (2.3).) In particular, $\mathcal{K}_{\Gamma}^{0}$ is the set of $C^{\infty}$ functions supported in $-\Gamma_{0}$.

Theorem 2.5 (Muramatu's integral formula). Let $1 \leq p<\infty, m \in \mathbb{N}_{0}, N \in \mathbb{N}$ and $\lambda>0$. Then there exist a function $\varphi \in \mathcal{K}_{\Gamma}^{0}$ satisfying $\int_{\Omega} \varphi(x) d x=1$, and functions $K_{\beta} \in \mathcal{K}_{\Gamma}^{N}$ with multi-indices $\beta$ satisfying $|\beta|=m$ such that every $f \in W_{p}^{m}(\Omega)$ is written as

$$
f=\int_{0}^{\lambda} \sum_{|\beta|=m} t^{m}\left(K_{\beta}\right)_{t} * \partial^{\beta} f \frac{d t}{t}+\varphi_{\lambda} * f
$$

where the integral $\int_{0}^{\lambda}$ should be interpreted as the limit of $\int_{\epsilon}^{\lambda}$ as $\epsilon \rightarrow 0$ in the norm of $L_{p}(\Omega)$. More generally,

$$
\partial^{\alpha} f=\int_{0}^{\lambda} \sum_{|\beta|=m} t^{m-|\alpha|}\left(K_{\beta}^{(\alpha)}\right)_{t} * \partial^{\beta} f \frac{d t}{t}+\lambda^{-|\alpha|}\left(\varphi^{(\alpha)}\right)_{\lambda} * f
$$

for $|\alpha| \leq m$.

Remark 2.6. Expression (2.15) implies that we may interchange the order of integration and differentiation, when applying $\partial^{\alpha}$ to $(2.14)$. We will discuss the problem of differentiation under integral sign in the other situations in Proposition 2.9 and Corollary 6.2. At this stage we may not interchange the order of integration and summation in Muramatu's formula, since it has not been guaranteed that the integral of each term in the sum converges in $L_{p}(\Omega)$. Proposition 2.9 and Theorem 6.1 will give criteria for the $L_{p}$ convergence of the integral. 
Proof. Take $\omega \in \mathcal{K}_{\Gamma}^{0}$ satisfying $\int_{\mathbb{R}^{d}} \omega(x) d x=1$, and define $\varphi$ and $K$ as in Lemma 2.3 with $N$ replaced by $N+m$. Then we find that $(2.11)$ is valid, since $\int_{\mathbb{R}^{d}} \varphi(x) d x=1$ by integration by parts. Applying (2.11) to $f^{(\alpha)} \in L_{p}(\Omega)$ with $|\alpha| \leq m$, we get

$$
f^{(\alpha)}=\int_{0}^{\lambda} K_{t} * f^{(\alpha)} \frac{d t}{t}+\varphi_{\lambda} * f^{(\alpha)} .
$$

In view of (2.13) with $N$ replaced by $N+m$ we know that $K$ is written as $K=$ $\sum_{|\beta|=m} K_{\beta}^{(\beta)}$ with some $K_{\beta} \in \mathcal{K}_{\Gamma}^{N}$, and hence

$$
f^{(\alpha)}=\int_{0}^{\lambda} \sum_{|\beta|=m}\left(K_{\beta}^{(\beta)}\right)_{t} * f^{(\alpha)} \frac{d t}{t}+\varphi_{\lambda} * f^{(\alpha)} .
$$

We note that in general

$$
\psi * f^{(\alpha)}=\left(\partial^{\alpha} \psi\right) * f
$$

holds for $\psi \in \mathcal{K}_{\Gamma}^{0}$. In fact, by the definition of distributional derivatives we have

$$
\begin{aligned}
\psi * f^{(\alpha)}(x) & =\int_{\Omega} \psi(x-y) f^{(\alpha)}(y) d y=\int_{\Omega}\left\{\left(-\partial_{y}\right)^{\alpha} \psi(x-y)\right\} f(y) d y \\
& =\int_{\Omega}\left\{\partial_{x}^{\alpha} \psi(x-y)\right\} f(y) d y .
\end{aligned}
$$

Using (2.16), we have $\varphi_{\lambda} * f^{(\alpha)}=\lambda^{-|\alpha|}\left(\varphi^{(\alpha)}\right)_{\lambda} * f$ and

$$
\left(K_{\beta}^{(\beta)}\right)_{t} * f^{(\alpha)}=t^{-|\alpha|}\left(K_{\beta}^{(\alpha+\beta)}\right)_{t} * f=t^{|\beta|-|\alpha|}\left(K_{\beta}^{(\alpha)}\right)_{t} * f^{(\beta)} .
$$

Therefore we get (2.15) with $K_{\beta}^{(\alpha)} \in \mathcal{K}_{\Gamma}^{N+|\alpha|} \subset \mathcal{K}_{\Gamma}^{N}$.

As an application of Muramatu's integral formula we show the interpolation inequality. We use the symbol

$$
\left\|\nabla^{m} f\right\|_{L_{p}(\Omega)}=\sum_{|\alpha|=m}\left\|\partial^{\alpha} f\right\|_{L_{p}(\Omega)} .
$$

Theorem 2.7 (Interpolation inequality). Let $1 \leq p<\infty, m \in \mathbb{N}, k \in \mathbb{N}$ and $1 \leq k<$ $m$. Then there exists $C=C(m, p, d, \Omega)>0$ such that the inequalities

$$
\begin{aligned}
& \left\|\nabla^{k} f\right\|_{L_{p}(\Omega)} \leq C\left(\|f\|_{L_{p}(\Omega)}+\left\|\nabla^{m} f\right\|_{L_{p}(\Omega)}\right), \\
& \left\|\nabla^{k} f\right\|_{L_{p}(\Omega)} \leq C\|f\|_{L_{p}(\Omega)}^{1-k / m}\left\|\nabla^{m} f\right\|_{L_{p}(\Omega)}^{k / m}
\end{aligned}
$$

hold for $f \in W_{p}^{m}(\Omega)$.

Proof. We use Muramatu's formula $(2.15)$ for $f^{(\alpha)} \in L_{p}(\Omega)$ with $|\alpha|=k$. Minkowski's inequality and Young's inequality give

$$
\left\|f^{(\alpha)}\right\|_{L_{p}} \leq \int_{0}^{\lambda} \sum_{|\beta|=m} t^{m-k}\left\|K_{\beta}^{(\alpha)}\right\|_{L_{1}}\left\|f^{(\beta)}\right\|_{L_{p}} \frac{d t}{t}+\lambda^{-k}\left\|\varphi^{(\alpha)}\right\|_{L_{1}}\|f\|_{L_{p}},
$$

and thereby

$$
\left\|f^{(\alpha)}\right\|_{L_{p}} \leq C\left(\lambda^{m-k}\left\|\nabla^{m} f\right\|_{L_{p}}+\lambda^{-k}\|f\|_{L_{p}}\right) .
$$


We get (2.17) by setting $\lambda=1$. Inequality (2.18) follows by setting $\lambda=$ $\left(\|f\|_{L_{p}} /\left\|\nabla^{m} f\right\|_{L_{p}}\right)^{1 / m}$ so that $\lambda^{m-k}\left\|\nabla^{m} f\right\|_{L_{p}}=\lambda^{-k}\|f\|_{L_{p}}$ if $\left\|\nabla^{m} f\right\|_{L_{p}} \neq 0$. When $\left\|\nabla^{m} f\right\|_{L_{p}}=0$, letting $\lambda \rightarrow \infty$ in (2.19) yields $f^{(\alpha)}=0$, so (2.18) follows.

Remark 2.8. If $\Omega$ satisfies $\Omega=\lambda \Omega$ for all $\lambda>0$, then inequality (2.19) follows from

$$
\left\|f^{(\alpha)}\right\|_{L_{p}(\Omega)} \leq C\left(\|f\|_{L_{p}(\Omega)}+\left\|\nabla^{m} f\right\|_{L_{p}(\Omega)}\right) .
$$

by the scaling method. Indeed, we get (2.19) from (2.20) by replacing $f$ with $f(\lambda \cdot)$, the function $x \mapsto f(\lambda x)$, and using $\partial^{\alpha}\{f(\lambda \cdot)\}=\lambda^{|\alpha|} f^{(\alpha)}(\lambda \cdot)$ and $\|f(\lambda \cdot)\|_{L_{p}}=$ $\lambda^{-d / p}\|f\|_{L_{p}}$. One advantage of Muramatu's formula is that we can directly obtain (2.19) without passing through (2.20), and that there is no need to assume that $\Omega$ satisfies $\Omega=\lambda \Omega$ for all $\lambda>0$.

It is possible to relax the assumption in the interpolation inequality. To this end, we prepare a lemma on convergence in a simple case.

Proposition 2.9. Let $1 \leq p \leq \infty, 0<\epsilon<\lambda$. Suppose that a function $u(t, x)$ is in $C^{\infty}((0, \infty) \times \Omega)$, and that $\int_{0}^{\lambda}\|u(t, \cdot)\|_{L_{p}(\Omega)} d t<\infty$.

(i) The integral $\int_{\epsilon}^{\lambda} u(t, x) d t$, which is a function of $x$, converges in $L_{p}(\Omega)$ as $\epsilon \rightarrow 0$. If we write $\int_{0}^{\lambda} u(t, \cdot) d t$ for the limit, we have

$$
\left\|\int_{0}^{\lambda} u(t, \cdot) d t\right\|_{L_{p}(\Omega)} \leq \int_{0}^{\lambda}\|u(t, \cdot)\|_{L_{p}(\Omega)} d t .
$$

In particular, if $p=\infty$, then $\int_{0}^{\lambda} u(t, \cdot) d t \in C(\Omega)$.

(ii) In addition to the above assumption, suppose that

$$
\int_{0}^{\lambda}\left\|\partial^{\alpha} u(t, \cdot)\right\|_{L_{p}(\Omega)} d t<\infty
$$

for a multi-index $\alpha$ with $|\alpha|>0$, where $\partial^{\alpha} u(t, x)$ stands for $(\partial / \partial x)^{\alpha} u(t, x)$. Then both of $\partial^{\alpha} \int_{0}^{\lambda} u(t, \cdot) d t$ and $\int_{0}^{\lambda} \partial^{\alpha} u(t, \cdot) d t$ belong to $L_{p}(\Omega)$, and they are equal:

$$
\partial^{\alpha} \int_{0}^{\lambda} u(t, \cdot) d t=\int_{0}^{\lambda} \partial^{\alpha} u(t, \cdot) d t .
$$

Proof. The first half of (i) follows from Minkowski's inequality and the completeness of $L_{p}(\Omega)$. The assertion for $p=\infty$ follows from the fact that convergence in the $L_{\infty}$ norm implies uniform convergence. We get (ii) by (i) and Lemma 2.1, noting that $\partial^{\alpha} \int_{\epsilon}^{\lambda} u(t, \cdot) d t=\int_{\epsilon}^{\lambda} \partial^{\alpha} u(t, \cdot) d t$ for $0<\epsilon<\lambda$.

Corollary 2.10. Let $1 \leq p<\infty$ and $m \in \mathbb{N}$. Suppose that $f \in L_{p}(\Omega)$ and $\partial^{\alpha} f \in L_{p}(\Omega)$ for all $\alpha$ with $|\alpha|=m$. Then $f \in W_{p}^{m}(\Omega)$, and interpolation inequalities (2.17), (2.18) hold. 
Proof. From the proof of Theorem 2.5, we see that (2.14) and (2.15) with $|\alpha|=m$ are valid. In order to show that (2.15) is also valid for $0<|\alpha|<m$, we use (2.14). Observing

$$
\begin{aligned}
\int_{0}^{\lambda}\left\|\partial^{\alpha}\left\{t^{m}\left(K_{\beta}\right)_{t} * f^{(\beta)}\right\}\right\|_{L_{p}} \frac{d t}{t} & =\int_{0}^{\lambda} t^{m-|\alpha|-1}\left\|\left(K_{\beta}^{(\alpha)}\right)_{t} * f^{(\beta)}\right\|_{L_{p}} d t \\
& \leq\left\|K_{\beta}^{(\alpha)}\right\|_{L_{1}}\left\|f^{(\beta)}\right\|_{L_{p}} \int_{0}^{\lambda} t^{m-|\alpha|-1} d t<\infty
\end{aligned}
$$

for $|\alpha|<m$, and using Proposition 2.9, we get (2.15) for $|\alpha|<m$ by applying $\partial^{\alpha}$ to (2.14). Now that (2.15) holds for all $\alpha$ with $|\alpha| \leq m$, the proof of Theorem 2.7 works as it is.

We conclude this section with the theorem on approximation by $C^{\infty}$ functions.

Theorem 2.11. Let $1 \leq p<\infty$ and $m \in \mathbb{N}$. Then $W_{p}^{m}(\Omega) \cap C^{\infty}\left(\mathbb{R}^{d}\right)$ is dense in $W_{p}^{m}(\Omega)$. In other words, there exists a sequence $f_{n} \in W_{p}^{m}(\Omega) \cap C^{\infty}\left(\mathbb{R}^{d}\right)$ such that

$$
f_{n} \rightarrow f, \quad \text { in } W_{p}^{m}(\Omega) \text { as } n \rightarrow \infty .
$$

Proof. Take $\varphi \in C_{0}^{\infty}\left(\mathbb{R}^{d}\right)$ satisfying (2.4), and consider the convolution $\varphi_{t} * f$ defined by (2.5). Let $E_{0}$ be the zero-extension defined by (2.9). Then $\varphi_{t} * f$ is the restriction of $\varphi_{t} * E_{0} f \in C^{\infty}\left(\mathbb{R}^{d}\right)$ to $\Omega$. By definition of weak derivatives we have

$$
\partial^{\alpha}\left(\varphi_{t} * f\right)(x)=\left(\partial^{\alpha} \varphi_{t}\right) * f(x)=\varphi_{t} * f^{(\alpha)}(x), \quad \text { for } x \in \Omega,|\alpha| \leq m .
$$

Since $f^{(\alpha)} \in L_{p}(\Omega)$ for $|\alpha| \leq m$, we get $\partial^{\alpha}\left(\varphi_{t} * f\right) \rightarrow f^{(\alpha)}$ in $L_{p}(\Omega)$ as $t \rightarrow 0$ by (2.10). Therefore $\varphi_{t} * f \rightarrow f$ in $W_{p}^{m}(\Omega)$. Thus we obtain the theorem.

\section{Embeddings into other Sobolev spaces}

In this section we consider the embeddings such as $W_{p}^{m}(\Omega) \subset W_{q}^{n}(\Omega)$ with the Sobolev inequality

$$
\|f\|_{W_{q}^{n}(\Omega)} \leq C\|f\|_{W_{p}^{m}(\Omega)}, \quad \text { for } f \in W_{p}^{m}(\Omega) .
$$

We shall show by the scaling method that the conditions

$$
m-\frac{d}{p} \geq n-\frac{d}{q}, \quad p \leq q
$$

are required for inequality (3.1) to hold, assuming for a moment that $\Omega$ satisfies $\Omega=\lambda \Omega$ for all $\lambda>0$. Applying (3.1) to $f(\lambda \cdot)$, the function $x \mapsto f(\lambda x)$, instead of $f$ yields

$$
\sum_{|\beta| \leq n} \lambda^{|\beta|-d / q}\left\|\partial^{\beta} f\right\|_{L_{q}(\Omega)} \leq C \sum_{|\alpha| \leq m} \lambda^{|\alpha|-d / p}\left\|\partial^{\alpha} f\right\|_{L_{p}(\Omega)} .
$$

Compare the leading terms on the left and right sides as $\lambda \rightarrow \infty$. Since they are $\left\|\nabla^{n} f\right\|_{L_{q}} \lambda^{n-d / q}$ and $C\left\|\nabla^{m} f\right\|_{L_{p}} \lambda^{m-d / p}$, respectively, we must have $m-d / p \geq n-$ $d / q$. Since the leading terms as $\lambda \rightarrow 0$ on the left and right sides are $\|f\|_{L_{q}} \lambda^{-d / q}$ and $C\|f\|_{L_{p}} \lambda^{-d / p}$, respectively, we must have $-d / q \geq-d / p$, i.e. $p \leq q$. Thus we 
know that (3.2) is necessary for (3.1). Following Runst and Sickel [26], we call the quantity

$$
m-\frac{d}{p}
$$

which is the exponent appearing in the leading term of $\|f(\lambda \cdot)\|_{W_{p}^{m}}$ as $\lambda \rightarrow \infty$, the differential dimension of $W_{p}^{m}(\Omega)$. Generally, when a function space $X$ is continuously embedded into another function space $Y$, the differential dimension of $X$ is equal to or greater than that of $Y$.

Theorem 3.1. Let $1 \leq p<q<\infty, m \in \mathbb{N}, n \in \mathbb{N}_{0}$ and $m-d / p \geq n-d / q$. Then

$$
W_{p}^{m}(\Omega) \subset W_{q}^{n}(\Omega),
$$

and the inequality

$$
\|f\|_{W_{q}^{n}(\Omega)} \leq C\|f\|_{W_{p}^{m}(\Omega)}, \quad \text { for } f \in W_{p}^{m}(\Omega)
$$

holds with $C=C(m, n, p, q, d, \Omega)$. Furthermore,

$$
\left\|\nabla^{k} f\right\|_{L_{q}(\Omega)} \leq C\|f\|_{L_{p}(\Omega)}^{1-\frac{k}{m}-\frac{d}{m}\left(\frac{1}{p}-\frac{1}{q}\right)}\left\|\nabla^{m} f\right\|_{L_{p}(\Omega)}^{\frac{k}{m}+\frac{d}{m}\left(\frac{1}{p}-\frac{1}{q}\right)}, \quad \text { for } 0 \leq k \leq n .
$$

In particular, if $m-d / p=n-d / q$, then

$$
\left\|\nabla^{n} f\right\|_{L_{q}(\Omega)} \leq C\left\|\nabla^{m} f\right\|_{L_{p}(\Omega)}
$$

Proof. First of all we note that $m>n$, since $m-n \geq d\left(p^{-1}-q^{-1}\right)>0$. We define $r \in(1, \infty)$ so that $p^{-1}+r^{-1}=q^{-1}+1$.

Case 1: $m-d / p>n-d / q$. Let $\alpha \in \mathbb{N}_{0}^{d}$ and $k \in \mathbb{N}_{0}$ satisfy $|\alpha|=k \leq n$. We use Muramatu's formula (2.15) for $W_{p}^{m}(\Omega)$, and apply Proposition 2.9 for $L_{q}(\Omega)$. Minkowski's inequality and Young's inequality with $\left\|\left(K_{\beta}^{(\alpha)}\right)_{t}\right\|_{L_{r}}=t^{-d(1-1 / r)}\left\|K_{\beta}^{(\alpha)}\right\|_{L_{r}}$ give

$$
\begin{aligned}
\left\|f^{(\alpha)}\right\|_{L_{q}} \leq & \int_{0}^{\lambda} t^{m-k-d(1 / p-1 / q)} \sum_{|\beta|=m}\left\|K_{\beta}^{(\alpha)}\right\|_{L_{r}}\left\|f^{(\beta)}\right\|_{L_{p}} \frac{d t}{t} \\
& \quad+\lambda^{-k-d(1 / p-1 / q)}\left\|\varphi^{(\alpha)}\right\|_{L_{r}}\|f\|_{L_{p}} \\
\leq & C\left(\lambda^{m-k-(d / p-d / q)}\left\|\nabla^{m} f\right\|_{L_{p}}+\lambda^{-k-(d / p-d / q)}\|f\|_{L_{p}}\right)
\end{aligned}
$$

for $\lambda>0$, where we used $m-d / p>k-d / q$. Setting $\lambda=1$, we get (3.3) and (3.4). Inequality (3.5) follows by choosing $\lambda$ so that $\lambda^{m}\left\|\nabla^{m} f\right\|_{L_{p}}=\|f\|_{L_{p}}$ if $\left\|\nabla^{m} f\right\|_{L_{p}} \neq 0$. When $\left\|\nabla^{m} f\right\|_{L_{p}}=0$, letting $\lambda \rightarrow \infty$ yields $f^{(\alpha)}=0$, so (3.5) follows.

Case 2: $p>1$ and $m-d / p=n-d / q$. If $|\alpha|<n$, then we can evaluate $f^{(\alpha)}$ in the same way as in Case 1. So we need to evaluate $f^{(\alpha)}$ only for $|\alpha|=n$. In Muramatu's formula (2.15) we observe that $\left\|\lambda^{-|\alpha|}\left(\varphi^{(\alpha)}\right)_{\lambda} * f\right\|_{L_{\infty}} \leq \lambda^{-n-d / p}\left\|\varphi^{(\alpha)}\right\|_{L_{p^{\prime}}}\|f\|_{L_{p}} \rightarrow 0$ as $\lambda \rightarrow \infty$, where $1 / p+1 / p^{\prime}=1$, and note that the integral $\int_{0}^{\lambda}$ is the limit of $\int_{\epsilon}^{\lambda}$ in 
$L_{p}(\Omega)$ as $\epsilon \rightarrow 0$. Hence there exists a sequence of positive numbers $\left\{\epsilon_{j}\right\}_{j=1}^{\infty}$ tending to 0 such that

$$
f^{(\alpha)}(x)=\lim _{j \rightarrow \infty} \int_{\epsilon_{j}}^{\epsilon_{j}^{-1}} t^{m-n} \sum_{|\beta|=m}\left(K_{\beta}^{(\alpha)}\right)_{t} * f^{(\beta)}(x) \frac{d t}{t}, \quad \text { for a.e. } x \in \Omega,
$$

which gives

$$
\left|f^{(\alpha)}(x)\right| \leq C \int_{0}^{\infty} \frac{d t}{t} \int_{\Omega} t^{m-n-d} \chi\left(\frac{|x-y|}{t}\right) g(y) d y, \quad \text { for a.e. } x \in \Omega,
$$

where $g(x)=\sum_{|\beta|=m}\left|f^{(\beta)}(x)\right|$, and $\chi$ is the characteristic function of the interval $[0,1]$. Since $m-n-d=d\left(p^{-1}-q^{-1}-1\right)=-d / r<0$ and $\int_{0}^{\infty} t^{m-n-d} \chi(\mid x-$ $y \mid / t) t^{-1} d t=(r / d)|x-y|^{-d / r}$, Fubini's theorem gives

$$
\left|f^{(\alpha)}(x)\right| \leq C \int_{\Omega}|x-y|^{-d / r} g(y) d y, \quad \text { for a.e. } x \in \Omega .
$$

By the Hardy-Littlewood-Sobolev inequality, which is a special case of $[12$, Theorem 6.36], we obtain $\left\|f^{(\alpha)}\right\|_{L_{q}} \leq C\|g\|_{L_{p}} \leq C\left\|\nabla^{m} f\right\|_{L_{p}}$.

Case 3: $p=1$ and $m-d / p=n-d / q$. This case is outside the scope of Muramatu's formula. We may assume $\Omega=\mathbb{R}^{d}$ with the help of the extension operator given in Theorem 5' of [30, Chapter VI]. We refer to [9, Chapter 5], [30, Chapter V], $[34$, Chapter 13] for the proof of the case $p=m=1$, which implies $q=d /(d-1)$, $n=0$ and $d \geq 2$.

Let $p=1$ and $m \geq 2$, which imply $m-d=n-d / q$ and hence $d \geq 2$, since if $d=1$ were valid, then $m-n=1-1 / q$ would not be an integer, a contradiction. Let $f \in W_{1}^{m}\left(\mathbb{R}^{d}\right)$ and set $s=d /(d-1)$. Since $\partial^{\alpha} f \in W_{1}^{1}\left(\mathbb{R}^{d}\right)$ for $|\alpha| \leq m-1$, it follows from the result for $p=m=1$ that $\left\|\partial^{\alpha} f\right\|_{L_{s}} \leq C\left\|\nabla\left(\partial^{\alpha} f\right)\right\|_{L_{1}}$ for $|\alpha| \leq m-1$, and thereby $f \in W_{s}^{m-1}\left(\mathbb{R}^{d}\right)$ with $\|f\|_{W_{s}^{m-1}} \leq C\|f\|_{W_{1}^{m}}$. If $q=s$, then this is the desired result. If $q>s$, then we have $m-1-d / s=n-d / q, m-1>n$, and it follows from the result in Case 2 that $W_{s}^{m-1} \subset W_{q}^{n}\left(\mathbb{R}^{d}\right)$ with $\|f\|_{W_{q}^{n}} \leq C\|f\|_{W_{s}^{m-1}}$, which gives the desired result. Thus we complete the proof for $p=1$ and $m \geq 2$.

It is a good exercise to prove Nash's inequality by Muramatu's formula. Indeed, we can prove it in the same spirit as in the proof of Theorem 3.1 for the case $m-d / p>n-d / q$.

Theorem 3.2 (Nash's inequality). Let $f \in L_{1}\left(\mathbb{R}^{d}\right) \cap W_{2}^{1}\left(\mathbb{R}^{d}\right)$. Then $f \in L_{2}\left(\mathbb{R}^{d}\right)$ and

$$
\|f\|_{L_{2}\left(\mathbb{R}^{d}\right)} \leq C(d)\|f\|_{L_{1}\left(\mathbb{R}^{d}\right)}^{2 /(d+2)}\|\nabla f\|_{L_{2}\left(\mathbb{R}^{d}\right)}^{d /(d+2)} .
$$

\section{Embeddings into Hölder-Zygmund spaces}

When the differential dimension $m-d / p$ is large enough, the functions in $W_{p}^{m}(\Omega)$ are differentiable in the classical sense. To describe the statement precisely we introduce the Hölder-Zygmund space. 
Definition 4.1 (Hölder-Zygmund space). For $k \in \mathbb{N}_{0}$ set

$$
|f|_{k, \Omega}=\sum_{|\alpha|=k} \sup _{x \in \Omega}\left|\partial^{\alpha} f(x)\right| .
$$

For $h \in \mathbb{R}^{d}$ let

$$
\Omega_{1, h}=\{x \in \Omega: x+h \in \Omega\}, \quad \Omega_{2, h}=\{x \in \Omega: x+h \in \Omega, x+2 h \in \Omega\} .
$$

The difference operators $\Delta_{h}$ and $\Delta_{h}^{2}$ are defined by

$$
\begin{aligned}
& \Delta_{h} f(x)=f(x+h)-f(x), \quad \text { for } x \in \Omega_{1, h}, \\
& \Delta_{h}^{2} f(x)=\Delta_{h}\left(\Delta_{h} f\right)(x)=f(x+2 h)-2 f(x+h)+f(x), \quad \text { for } x \in \Omega_{2, h} .
\end{aligned}
$$

We define the seminorm $[f]_{\tau, \Omega}$ for $0<\tau \leq 1$ by

$$
[f]_{\tau, \Omega}=\sup _{h \neq 0} \frac{\left|\Delta_{h} f\right|_{0, \Omega_{1, h}}}{|h|^{\tau}}, \quad \text { if } 0<\tau<1 ; \quad[f]_{1, \Omega}=\sup _{h \neq 0} \frac{\left|\Delta_{h}^{2} f\right|_{0, \Omega_{2, h}}}{|h|},
$$

where $\left|\Delta_{h} f\right|_{0, \Omega_{1, h}}$ should be interpreted as 0 if $\Omega_{1, h}$ is empty; similarly for $\left|\Delta_{h}^{2} f\right|_{0, \Omega_{2, h}}$. Let $\sigma>0$ and define $[\sigma]_{-}$and $\{\sigma\}_{+}$so that

$$
\sigma=[\sigma]_{-}+\{\sigma\}_{+}, \quad[\sigma]_{-} \in \mathbb{N}_{0}, \quad 0<\{\sigma\}_{+} \leq 1 .
$$

Then the Hölder-Zygmund space $\mathcal{C}^{\sigma}(\Omega)$ is the collection of all functions $f$ which are $[\sigma]_{-}$times continuously differentiable and satisfy

$$
\|f\|_{\mathcal{C}^{\sigma}(\Omega)}:=\sum_{|\alpha| \leq[\sigma]_{-}}\left|\partial^{\alpha} f\right|_{0, \Omega}+\sum_{|\alpha|=[\sigma]_{-}}\left[\partial^{\alpha} f\right]_{\{\sigma\}_{+}, \Omega}<\infty .
$$

We equip $\mathcal{C}^{\sigma}(\Omega)$ with the norm $\|\cdot\|_{\mathcal{C}^{\sigma}(\Omega)}$.

The Hölder-Zygmund space $\mathcal{C}^{\sigma}(\Omega)$ is a Banach space.

We shall find necessary conditions for $m \in \mathbb{N}, 1 \leq p<\infty$ and $\sigma>0$ that guarantee the embedding $W_{p}^{m}(\Omega) \subset \mathcal{C}^{\sigma}(\Omega)$ with the ineqaulity

$$
\|f\|_{\mathcal{C}^{\sigma}(\Omega)} \leq C\|f\|_{W_{p}^{m}(\Omega)}
$$

assuming for a moment that $\Omega$ satisfies $\Omega=\lambda \Omega$ for all $\lambda>0$. Applying (4.3) to $f(\lambda \cdot)$, the function $x \mapsto f(\lambda x)$, instead of $f$ yields

$$
\sum_{|\alpha| \leq[\sigma]_{-}} \lambda^{|\alpha|}\left|\partial^{\alpha} f\right|_{0, \Omega}+\lambda^{\sigma}[f]_{\sigma, \Omega} \leq C \sum_{|\alpha| \leq m} \lambda^{|\alpha|-d / p}\left\|\partial^{\alpha} f\right\|_{L_{p}(\Omega)}
$$

with $[f]_{\sigma, \Omega}=\sum_{|\alpha|=[\sigma]_{-}}\left[\partial^{\alpha} f\right]_{\{\sigma\}_{+}, \Omega}$. Since the leading terms as $\lambda \rightarrow \infty$ on the left and right sides are $[f]_{\sigma, \Omega} \lambda^{\sigma}$ and $\left\|\nabla^{m} f\right\|_{L_{p}} \lambda^{m-d / p}$, respectively, we must have

$$
\sigma \leq m-d / p \text {. }
$$

So this inequality is a necessary condition for (4.3). In view of the above argument it is reasonable to call $\sigma$, which is the exponent appearing in the leading term of $\|f(\lambda \cdot)\|_{\mathcal{C}^{\sigma}(\Omega)}$, the differential dimension of the Hölder-Zygmund space $\mathcal{C}^{\sigma}(\Omega)$. 
Theorem 4.2. Let $1 \leq p<\infty, m \in \mathbb{N}, m-d / p>0$, and set $\sigma=m-d / p$. Then

$$
W_{p}^{m}(\Omega) \subset \mathcal{C}^{\sigma}(\Omega)
$$

and the inequality

$$
\|f\|_{\mathcal{C}^{\sigma}(\Omega)} \leq C\|f\|_{W_{p}^{m}(\Omega)}, \quad \text { for } f \in W_{p}^{m}(\Omega)
$$

holds with $C=C(m, p, d, \Omega)$. Furthermore,

$$
\left|\partial^{\alpha} f\right|_{0, \Omega} \leq C\|f\|_{L_{p}(\Omega)}^{1-\frac{|\alpha|}{m}-\frac{d}{m p}}\left\|\nabla^{m} f\right\|_{L_{p}(\Omega)}^{\frac{|\alpha|}{m}+\frac{d}{m p}}, \quad \text { for } 0 \leq|\alpha| \leq[\sigma]_{-},
$$

and

$$
\left[\partial^{\alpha} f\right]_{\{\sigma\}_{+}, \Omega} \leq C\left\|\nabla^{m} f\right\|_{L_{p}(\Omega)}, \quad \text { for }|\alpha|=[\sigma]_{-} .
$$

Proof. Step 1. Let $0 \leq|\alpha| \leq[\sigma]_{-}$. We use Muramatu's formula (2.15). Then Hölder's inequality with $1 / p+1 / p^{\prime}=1$ gives

$$
\begin{aligned}
\left|f^{(\alpha)}(x)\right| \leq & \int_{0}^{\lambda} \sum_{|\beta|=m} t^{m-|\alpha|-d / p}\left\|K_{\beta}^{(\alpha)}\right\|_{L_{p^{\prime}}}\left\|f^{(\beta)}\right\|_{L_{p}} \frac{d t}{t} \\
& +\lambda^{-|\alpha|-d / p}\left\|\varphi^{(\alpha)}\right\|_{L_{p^{\prime}}}\|f\|_{L_{p}} \\
\leq & C\left(\lambda^{m-|\alpha|-d / p}\left\|\nabla^{m} f\right\|_{L_{p}}+\lambda^{-|\alpha|-d / p}\|f\|_{L_{p}}\right) .
\end{aligned}
$$

By Proposition 2.9 we have $f^{(\alpha)} \in C(\Omega)$ and thereby $f \in C^{[\sigma]}-(\Omega)$ with $\left|f^{(\alpha)}\right|_{0, \Omega} \leq$ $C\|f\|_{W_{p}^{m}(\Omega)}$. Inequality (4.5) follows by optimizing the sum in the parentheses.

Step 2. Next we evaluate the Hölder seminorm of $f^{(\alpha)}$ for $|\alpha|=[\sigma]_{-}$, assuming $0<\{\sigma\}_{+}<1$. Set $k=[\sigma]_{-}$and $\tau=\{\sigma\}_{+}$. With

$$
\lambda^{-|\alpha|}\left\|\left(\varphi^{(\alpha)}\right)_{\lambda} * f\right\|_{L_{\infty}} \leq \lambda^{-|\alpha|-d / p}\left\|\varphi^{(\alpha)}\right\|_{L_{p^{\prime}}}\|f\|_{L_{p}}
$$

in mind, we let $\lambda \rightarrow \infty$ in Muramatu's formula (2.15) to get

$$
f^{(\alpha)}=\int_{0}^{\infty} \sum_{|\beta|=m} t^{m-k}\left(K_{\beta}^{(\alpha)}\right)_{t} * f^{(\beta)} \frac{d t}{t},
$$

where the integral $\int_{0}^{\infty}$ should be interpreted as the limit of $\int_{\epsilon}^{\lambda}$ as $\epsilon \rightarrow 0$ and $\lambda \rightarrow \infty$ in the topology of $L_{p}(\Omega)+L_{\infty}(\Omega)$. We use the inequality

$$
\left\|\Delta_{h} K_{t}\right\|_{L_{p^{\prime}}\left(\mathbb{R}^{d}\right)} \leq C t^{-d / p} \min \left\{1,|h| t^{-1}\right\}
$$

with $C=\max \left\{2\|K\|_{L_{p^{\prime}}},\|\nabla K\|_{L_{p^{\prime}}}\right\}$ for $h \in \mathbb{R}^{d}$ and $K \in C_{0}^{\infty}\left(\mathbb{R}^{d}\right)$. This inequality is obtained from $\left\|\Delta_{h} K_{t}\right\|_{L_{p^{\prime}}} \leq 2\left\|K_{t}\right\|_{L_{p^{\prime}}}$ and $\Delta_{h} K_{t}(x)=t^{-d-1} h \cdot \int_{0}^{1} \nabla K((x+\theta h) / t) d \theta$. 
Hölder's inequality and the change of variables $t=|h| s$ give

$$
\begin{aligned}
\left\|\Delta_{h} f^{(\alpha)}\right\|_{L_{\infty}\left(\Omega_{1, h}\right)} & \leq \int_{0}^{\infty} \sum_{|\beta|=m} t^{m-k}\left\|\Delta_{h}\left(K_{\beta}^{(\alpha)}\right)_{t} * f^{(\beta)}\right\|_{L_{\infty}\left(\Omega_{1, h}\right)} \frac{d t}{t} \\
& \leq C\left\|\nabla^{m} f\right\|_{L_{p}(\Omega)} \int_{0}^{\infty} t^{m-k-d / p} \min \left\{1,|h| t^{-1}\right\} \frac{d t}{t} \\
& \leq C\left\|\nabla^{m} f\right\|_{L_{p}(\Omega)}|h|^{\tau} \int_{0}^{\infty} s^{\tau} \min \left\{1, s^{-1}\right\} \frac{d s}{s} .
\end{aligned}
$$

Since the last integral is finite, we obtain $f \in \mathcal{C}^{\sigma}(\Omega)$ with (4.6). Also, (4.4) follows from (4.6) and the estimates obtained in Step 1.

Step 3. When $\{\sigma\}_{+}=1$, we can make the same argument as in Step 2 if we replace $\Delta_{h}$ by $\Delta_{h}^{2}$ and use, instead of (4.7),

$$
\left\|\Delta_{h}^{2} K_{t}\right\|_{L_{p^{\prime}}\left(\mathbb{R}^{d}\right)} \leq C t^{-d / p} \min \left\{1,|h|^{2} t^{-2}\right\},
$$

which follows by Taylor's formula.

\section{Trace theorem}

In this section we consider the trace or the restriction to a hyperplane of a function belonging to Sobolev spaces. To this end, we first give an intrinstic definition of the Besov space. It is convenient to use the symbols

$$
L_{q}^{*}\left(\mathbb{R}^{d}\right)=L_{q}\left(\mathbb{R}^{d},|h|^{-d} d h\right), \quad L_{q}^{*}\left(\mathbb{R}_{+}\right)=L_{q}\left(\mathbb{R}_{+}, t^{-1} d t\right)
$$

for $1 \leq q \leq \infty$ and $\mathbb{R}_{+}=(0, \infty)$. Recall that $\Omega_{1, h}$ and $\Omega_{2, h}$ are defined by (4.1).

Definition 5.1 (Besov space). Let $1 \leq p<\infty$ and $1 \leq q \leq \infty$. We define the seminorm $|f|_{B_{p q}^{\tau}(\Omega)}$ for $0<\tau \leq 1$ by

$$
|f|_{B_{p q}^{\tau}(\Omega)}=\left\|\frac{\left\|\Delta_{h} f\right\|_{L_{p}\left(\Omega_{1, h}\right)}}{|h|^{\tau}}\right\|_{L_{q}^{*}\left(\mathbb{R}^{d}\right)}=\left\{\int_{\mathbb{R}^{d}}\left(\frac{\left\|\Delta_{h} f\right\|_{L_{p}\left(\Omega_{1, h}\right)}}{|h|^{\tau}}\right)^{q} \frac{d h}{|h|^{d}}\right\}^{1 / q}
$$

with the usual modification for $q=\infty$ if $0<\tau<1$, and

$$
|f|_{B_{p q}^{1}(\Omega)}=\left\|\frac{\left\|\Delta_{h}^{2} f\right\|_{L_{p}\left(\Omega_{2, h}\right)}}{|h|}\right\|_{L_{q}^{*}\left(\mathbb{R}^{d}\right)}
$$

if $\tau=1$. Let $\sigma>0$ and define $[\sigma]_{-} \in \mathbb{N}_{0}$ and $\{\sigma\}_{+} \in(0,1]$ as in (4.2). Set

$$
|f|_{B_{p q}^{\sigma}(\Omega)}=\sum_{|\alpha|=[\sigma]_{-}}\left|\partial^{\alpha} f\right|_{B_{p q}^{\{\sigma\}_{+}}(\Omega)},
$$

which is consistent with the above definition for $|f|_{B_{p q}^{\tau}(\Omega)}$. The Besov space $B_{p q}^{\sigma}(\Omega)$ is defined by

$$
B_{p q}^{\sigma}(\Omega)=\left\{f \in W_{p}^{[\sigma]-}(\Omega):|f|_{B_{p q}^{\sigma}(\Omega)}<\infty\right\},
$$


and equipped with the norm

$$
\|f\|_{B_{p q}^{\sigma}(\Omega)}=\sum_{|\alpha| \leq[\sigma]_{-}}\left\|\partial^{\alpha} f\right\|_{L_{p}(\Omega)}+|f|_{B_{p q}^{\sigma}(\Omega)} .
$$

The Besov space $B_{p q}^{\sigma}(\Omega)$ is a Banach space. By the same argument as in the case of Sobolev spaces we find that it is reasonable to call $\sigma-d / p$ the differential dimension of $B_{p q}^{\sigma}(\Omega)$.

We will give a statement of the trace theorem only when the domain is the half space

$$
\mathbb{R}_{+}^{d}=\left\{x=\left(x^{\prime}, x_{d}\right): x^{\prime} \in \mathbb{R}^{d-1}, x_{d}>0\right\} .
$$

We identify $\mathbb{R}^{d-1}$ with $\partial \mathbb{R}_{+}^{d}=\left\{\left(x^{\prime}, 0\right): x^{\prime} \in \mathbb{R}^{d-1}\right\}$, the boundary of $\mathbb{R}_{+}^{d}$. The trace operator $\operatorname{Tr}$ is defined by

$$
\operatorname{Tr} f\left(x^{\prime}\right)=f\left(x^{\prime}, 0\right)
$$

for a continuous function $f$ on the closure of $\mathbb{R}_{+}^{d}$. In the trace theorem we need the Besov space $B_{p q}^{\sigma}\left(\mathbb{R}^{d-1}\right)$ with $p=q$.

Theorem 5.2. Let $1<p<\infty, m \in \mathbb{N}$ and $d \geq 2$. Then the trace operator $\operatorname{Tr}:$ $W_{p}^{m}\left(\mathbb{R}_{+}^{d}\right) \cap C^{\infty}\left(\overline{\mathbb{R}_{+}^{d}}\right) \rightarrow C^{\infty}\left(\mathbb{R}^{d-1}\right)$ extends to a bounded linear operator

$$
W_{p}^{m}\left(\mathbb{R}_{+}^{d}\right) \rightarrow B_{p p}^{m-1 / p}\left(\mathbb{R}^{d-1}\right),
$$

and the inequality

$$
\|\operatorname{Tr} f\|_{B_{p p}^{m-1 / p}\left(\mathbb{R}^{d-1}\right)} \leq C\|f\|_{W_{p}^{m}\left(\mathbb{R}_{+}^{d}\right)}, \quad \text { for } f \in W_{p}^{m}\left(\mathbb{R}_{+}^{d}\right)
$$

holds with $C=C(m, p, d)$. In particular,

$$
|\operatorname{Tr} f|_{B_{p p}^{m-1 / p}\left(\mathbb{R}^{d-1}\right)} \leq C\left\|\nabla^{m} f\right\|_{L_{p}\left(\mathbb{R}_{+}^{d}\right)} .
$$

We note that the differential dimension of $B_{p p}^{m-1 / p}\left(\mathbb{R}^{d-1}\right)$ coincides with that of $W_{p}^{m}\left(\mathbb{R}_{+}^{d}\right)$, i.e. $(m-1 / p)-(d-1) / p=m-d / p$. In the proof of Theorem 5.2 we use the lemma on boundedness of integral operators.

Lemma 5.3. Let $1 \leq q \leq \infty$ and $K \in L_{1}^{*}\left(\mathbb{R}_{+}\right)$. Then the integral operators

$$
f(t) \mapsto \int_{0}^{\infty} K\left(\frac{|h|}{t}\right) f(t) \frac{d t}{t}, \quad g(h) \mapsto \int_{\mathbb{R}^{d}} K\left(\frac{|h|}{t}\right) g(h) \frac{d h}{|h|^{d}},
$$

are bounded linear operators $L_{q}^{*}\left(\mathbb{R}_{+}\right) \rightarrow L_{q}^{*}\left(\mathbb{R}^{d}\right)$ and $L_{q}^{*}\left(\mathbb{R}^{d}\right) \rightarrow L_{q}^{*}\left(\mathbb{R}_{+}\right)$, respectively. The operator norms are bounded by $C(d)\|K\|_{L_{1}^{*}\left(\mathbb{R}_{+}\right)}$. The statement is also valid if we replace the integral kernel $K(|h| / t)$ by $K(t /|h|)$. When $d=1$, the assertion remains valid if we replace $\mathbb{R}^{d}$ and $L_{q}\left(\mathbb{R}^{d}\right)$ by $\mathbb{R}_{+}$and $L_{q}\left(\mathbb{R}_{+}\right)$, respectively.

Proof. The assertion for $f(t)$ is valid by the theorem about boundedness of integral operators (see [12, Theorem 6.18]), since

$$
\sup _{h \in \mathbb{R}^{d}} \int_{\mathbb{R}_{+}} K\left(\frac{|h|}{t}\right) \frac{d t}{t}=\omega_{d-1}^{-1} \sup _{t \in \mathbb{R}_{+}} \int_{\mathbb{R}^{d}} K\left(\frac{|h|}{t}\right) \frac{d h}{|h|^{d}}=\|K\|_{L_{1}^{*}\left(\mathbb{R}_{+}\right)}<\infty,
$$

where $\omega_{d-1}$ is the area of the unit sphere in $\mathbb{R}^{d}$. The other cases can be proved similarly. 
Proof. Step 1. Since $W_{p}^{m}\left(\mathbb{R}_{+}^{d}\right) \cap C^{\infty}\left(\overline{\mathbb{R}_{+}^{d}}\right)$ is dense in $W_{p}^{m}\left(\mathbb{R}_{+}^{d}\right)$ by Theorem 2.11 , we need only show (5.2) and (5.3), assuming that $f \in W_{p}^{m}\left(\mathbb{R}_{+}^{d}\right) \cap C^{\infty}\left(\overline{\mathbb{R}_{+}^{d}}\right)$.

Let $\alpha=\left(\alpha^{\prime}, 0\right) \in \mathbb{N}_{0}^{d}$ with $\alpha^{\prime} \in \mathbb{N}_{0}^{d-1}$ and $0 \leq\left|\alpha^{\prime}\right|<m$. We use Muramatu's formula (2.15). Since $f^{(\beta)} \in C\left(\overline{\mathbb{R}_{+}^{d}}\right)$ for $|\beta|=m$, and since

$$
\varphi_{\epsilon} * f^{(\beta)}(x)-f^{(\beta)}(x)=\int_{\Gamma_{0}} \varphi(-z)\left\{f^{(\beta)}(x+\epsilon z)-f^{(\beta)}(x)\right\} d z
$$

for $x \in \overline{\mathbb{R}_{+}^{d}}$, the integral in $(2.15)$ can be also regarded as the pointwise limit of $\int_{\epsilon}^{\lambda}$ as $\epsilon \rightarrow 0$ on $\overline{\mathbb{R}_{+}^{d}}$. So we have

$$
\begin{aligned}
& (\operatorname{Tr} f)^{\left(\alpha^{\prime}\right)}\left(x^{\prime}\right)=f^{(\alpha)}\left(x^{\prime}, 0\right) \\
& =\int_{0}^{\lambda} \sum_{|\beta|=m} t^{m-|\alpha|}\left(K_{\beta}^{(\alpha)}\right)_{t} * f^{(\beta)}\left(x^{\prime}, 0\right) \frac{d t}{t}+\lambda^{-|\alpha|}\left(\varphi^{(\alpha)}\right)_{\lambda} * f\left(x^{\prime}, 0\right) .
\end{aligned}
$$

We write

$$
\left(K_{\beta}^{(\alpha)}\right)_{t} * f^{(\beta)}\left(x^{\prime}, 0\right)=\int_{0}^{\infty} d y_{d} \int_{\mathbb{R}^{d-1}}\left(K_{\beta}^{(\alpha)}\right)_{t}\left(x^{\prime}-y^{\prime},-y_{d}\right) f^{(\beta)}\left(y^{\prime}, y_{d}\right) d y^{\prime}
$$

with $y=\left(y^{\prime}, y_{d}\right)$, and use the inequality

$$
\left|\left(K_{\beta}^{(\alpha)}\right)_{t}(x)\right| \leq\left\|K_{\beta}^{(\alpha)}\right\|_{L_{\infty}} t^{-d} \chi\left(\left|x^{\prime}\right| / t\right) \chi\left(\left|x_{d}\right| / t\right),
$$

where $\chi$ is the characteristic function of $[0,1]$. Setting $\lambda=1$, and using Minkowski's inequality with Young's inequality, we have

$$
\begin{aligned}
& \left\|(\operatorname{Tr} f)^{\left(\alpha^{\prime}\right)}\right\|_{L_{p}\left(\mathbb{R}^{d-1}\right)} \\
& \leq C \sum_{|\beta|=m} \int_{0}^{1} \frac{d t}{t} \int_{0}^{\infty} t^{m-|\alpha|-1} \chi\left(\frac{y_{d}}{t}\right)\left\|f^{(\beta)}\left(\cdot, y_{d}\right)\right\|_{L_{p}\left(\mathbb{R}^{d-1}\right)} d y_{d} \\
& \quad+C \int_{0}^{\infty} \chi\left(y_{d}\right)\left\|f\left(\cdot, y_{d}\right)\right\|_{L_{p}\left(\mathbb{R}^{d-1}\right)} d y_{d} .
\end{aligned}
$$

Since $\|\chi(\cdot / t)\|_{L_{p^{\prime}}\left(\mathbb{R}_{+}\right)}=t^{1-1 / p}$ with $1 / p+1 / p^{\prime}=1$, Hölder's inequality gives

$$
\left\|(\operatorname{Tr} f)^{\left(\alpha^{\prime}\right)}\right\|_{L_{p}\left(\mathbb{R}^{d-1}\right)} \leq C\left\|\nabla^{m} f\right\|_{L_{p}\left(\mathbb{R}_{+}^{d}\right)} \int_{0}^{1} t^{m-|\alpha|-1 / p} \frac{d t}{t}+C\|f\|_{L_{p}\left(\mathbb{R}_{+}^{d}\right)} .
$$

In view of $m-|\alpha|-1 / p \geq 1-1 / p>0$ we find that $\operatorname{Tr} f \in W_{p}^{m-1}\left(\mathbb{R}^{d-1}\right)$ and $\|\operatorname{Tr} f\|_{W_{p}^{m-1}\left(\mathbb{R}^{d-1}\right)} \leq C\|f\|_{W_{p}^{m}\left(\mathbb{R}_{+}^{d}\right)}$.

Step 2. Next we shall evaluate the seminorms $\left|\partial^{\alpha} f\right|_{B_{p p}^{1-1 / p}\left(\mathbb{R}^{d-1}\right)}$ for $\alpha=\left(\alpha^{\prime}, 0\right)$ with $\alpha^{\prime} \in \mathbb{N}_{0}^{d-1}$ and $|\alpha|=\left|\alpha^{\prime}\right|=m-1$. Since the same calculations as in Step 1 give

$$
\lambda^{-|\alpha|}\left\|\left(\varphi^{(\alpha)}\right)_{\lambda} * f(\cdot, 0)\right\|_{L_{p}\left(\mathbb{R}^{d-1}\right)} \leq C \lambda^{-m+1-1 / p}\|f\|_{L_{p}\left(\mathbb{R}_{+}^{d}\right)},
$$

letting $\lambda \rightarrow \infty$ in (5.4) gives

$$
(\operatorname{Tr} f)^{\left(\alpha^{\prime}\right)}\left(x^{\prime}\right)=\int_{0}^{\infty} \sum_{|\beta|=m}\left(K_{\beta}^{(\alpha)}\right)_{t} * f^{(\beta)}\left(x^{\prime}, 0\right) d t .
$$


Let $h \in \mathbb{R}^{d-1}$. Evaluating $\left\|\left(K_{\beta}^{(\alpha)}\right)_{t}\left(\cdot+h, y_{d}\right)-\left(K_{\beta}^{(\alpha)}\right)_{t}\left(\cdot, y_{d}\right)\right\|_{L_{\infty}}$ by Taylor's formula, and taking into account the support of $K_{\beta}^{(\alpha)}$, we have

$$
\begin{aligned}
& \left|\left(K_{\beta}^{(\alpha)}\right)_{t}\left(x^{\prime}+h-y, y_{d}\right)-\left(K_{\beta}^{(\alpha)}\right)_{t}\left(x^{\prime}-y^{\prime}, y_{d}\right)\right| \\
& \leq C t^{-d} \min \left\{1,|h| t^{-1}\right\}\left\{\chi\left(\frac{\left|x^{\prime}+h-y^{\prime}\right|}{t}\right)+\chi\left(\frac{\left|x^{\prime}-y^{\prime}\right|}{t}\right)\right\} \chi\left(\frac{y_{d}}{t}\right) .
\end{aligned}
$$

Setting $u\left(y_{d}\right)=\sum_{|\beta|=m}\left\|f^{(\beta)}\left(\cdot, y_{d}\right)\right\|_{L_{p}\left(\mathbb{R}^{d-1}\right)}$, and using Minkowski's inequality with Young's inequality, we have

$$
\left\|\Delta_{h}(\operatorname{Tr} f)^{\left(\alpha^{\prime}\right)}\right\|_{L_{p}\left(\mathbb{R}^{d-1}\right)} \leq C \int_{0}^{\infty} \frac{d t}{t} \int_{0}^{\infty} \min \left\{1,|h| t^{-1}\right\} \chi\left(\frac{y_{d}}{t}\right) u\left(y_{d}\right) d y_{d} .
$$

Changing the variables $y_{d} \rightarrow s$ and $t \rightarrow|h| t$, and setting

$$
H(s)=\int_{0}^{\infty} s^{1-1 / p} \min \left\{1, t^{-1}\right\} \chi\left(\frac{s}{t}\right) \frac{d t}{t},
$$

we get

$$
\frac{\left\|\Delta_{h}(\operatorname{Tr} f)^{\left(\alpha^{\prime}\right)}\right\|_{L_{p}\left(\mathbb{R}^{d-1}\right)}}{|h|^{1-1 / p}} \leq C \int_{0}^{\infty} H\left(\frac{s}{|h|}\right)\left\{s^{1 / p} u(s)\right\} \frac{d s}{s}:=F(h) .
$$

Since

$$
\int_{0}^{\infty} H(s) \frac{d s}{s}=\int_{0}^{\infty} \frac{t^{1-1 / p}}{1-p^{-1}} \min \left\{1, t^{-1}\right\} \frac{d t}{t}<\infty,
$$

the map $s^{1 / p} u(s) \mapsto F(h)$ is a bounded operator from $L_{p}^{*}\left(\mathbb{R}_{+}\right)$to $L_{p}^{*}\left(\mathbb{R}^{d-1}\right)$ by Lemma 5.3. In view of $\left\|s^{1 / p} u(s)\right\|_{L_{p}^{*}\left(\mathbb{R}_{+}\right)}=\|u\|_{L_{p}\left(\mathbb{R}_{+}\right)} \leq\left\|\nabla^{m} f\right\|_{L_{p}\left(\mathbb{R}_{+}^{d}\right)}$ we obtain

$$
\left|(\operatorname{Tr} f)^{\left(\alpha^{\prime}\right)}\right|_{B_{p p}^{1-1 / p}\left(\mathbb{R}^{d-1}\right)} \leq C\left\|\nabla^{m} f\right\|_{L_{p}\left(\mathbb{R}_{+}^{d}\right)},
$$

which implies (5.3). Combining the result in Step 1, we conclude that $\operatorname{Tr} f \in$ $B_{p p}^{m-1 / p}\left(\mathbb{R}^{d-1}\right)$ with (5.2).

Corollary 5.4. The trace operator $\operatorname{Tr}: W_{p}^{m}\left(\mathbb{R}_{+}^{d}\right) \rightarrow B_{p p}^{m-1 / p}\left(\mathbb{R}^{d-1}\right)$ given in Theorem 5.2 is surjective.

Proof. Step 1. We shall evaluate the $L_{p}$ norm of the function $v(x)$ defined by

$$
v(x)=x_{d}^{-1} \psi_{x_{d}} * g\left(x^{\prime}\right)=\int_{\mathbb{R}^{d-1}} x_{d}^{-d} \psi\left(\frac{x^{\prime}-y^{\prime}}{x_{d}}\right) g\left(y^{\prime}\right) d y^{\prime}
$$

for $g \in B_{p p}^{1-1 / p}\left(\mathbb{R}^{d-1}\right)$ and $\psi \in C_{0}^{\infty}\left(\mathbb{R}^{d-1}\right)$ satisfying $\int_{\mathbb{R}^{d-1}} \psi\left(x^{\prime}\right) d x^{\prime}=0, \operatorname{supp} \psi \subset$ $\left\{x^{\prime}:\left|x^{\prime}\right|<1\right\}$. Rewriting $v$ as

$$
v\left(x^{\prime}, x_{d}\right)=\int_{\mathbb{R}^{d-1}} x_{d}^{-d} \psi\left(\frac{-y^{\prime}}{x_{d}}\right)\left\{g\left(x^{\prime}+y^{\prime}\right)-g\left(x^{\prime}\right)\right\} d y^{\prime},
$$


and using Minkowski's inequality, we have

$$
\begin{aligned}
& x_{d}^{1 / p}\left\|v\left(\cdot, x_{d}\right)\right\|_{L_{p}\left(\mathbb{R}^{d-1}\right)} \\
& \leq C \int_{\mathbb{R}^{d-1}}\left(\frac{\left|y^{\prime}\right|}{x_{d}}\right)^{d-1 / p} \chi\left(\frac{\left|y^{\prime}\right|}{x_{d}}\right) \frac{\left\|\Delta_{y^{\prime}} g\right\|_{L_{p}\left(\mathbb{R}^{d-1}\right)}}{\left|y^{\prime}\right|^{1-1 / p}} \frac{d y^{\prime}}{\left|y^{\prime}\right|^{d-1}},
\end{aligned}
$$

where $\chi$ is the characteristic function of $[0,1]$. Since $\int_{0}^{\infty} t^{d-1 / p} \chi(t) t^{-1} d t=1 /(d-$ $\left.p^{-1}\right)<\infty$, the map

$$
\left\|\Delta_{y^{\prime}} g\right\|_{L_{p}\left(\mathbb{R}^{d-1}\right)} /\left|y^{\prime}\right|^{1-1 / p} \mapsto x_{d}^{1 / p}\left\|v\left(\cdot, x_{d}\right)\right\|_{L_{p}\left(\mathbb{R}^{d-1}\right)}
$$

is a bounded operator from $L_{p}^{*}\left(\mathbb{R}^{d-1}\right)$ to $L_{p}^{*}\left(\mathbb{R}_{+}\right)$by Lemma 5.3. Therefore we get

$$
\|v\|_{L_{p}\left(\mathbb{R}_{+}^{d}\right)}=\left\|x_{d}^{1 / p}\right\| v\left(\cdot, x_{d}\right)\left\|_{L_{p}\left(\mathbb{R}^{d-1}\right)}\right\|_{L_{p}\left(\mathbb{R}_{+}\right)} \leq C|g|_{B_{p p}^{1-1 / p}\left(\mathbb{R}^{d-1}\right)} .
$$

Step 2. We shall evaluate the $L_{p}$ norm of the higher-order derivatives of $u$ defined by

$$
u(x)=\varphi_{x_{d}} * g\left(x^{\prime}\right)=\int_{\mathbb{R}^{d-1}} x_{d}^{1-d} \varphi\left(\frac{x^{\prime}-y^{\prime}}{x_{d}}\right) g\left(y^{\prime}\right) d y^{\prime}
$$

for $g \in B_{p p}^{m-1 / p}\left(\mathbb{R}^{d-1}\right)$ and $\varphi \in C_{0}^{\infty}\left(\mathbb{R}^{d-1}\right)$ satisfying $\int_{\mathbb{R}^{d-1}} \varphi\left(x^{\prime}\right) d x^{\prime}=1, \operatorname{supp} \varphi \subset$ $\left\{x^{\prime}:\left|x^{\prime}\right|<1\right\}$. We have $\partial_{j} u(x)=\varphi_{x_{d}} * \partial_{j} g\left(x^{\prime}\right)$ for $1 \leq j<d$ and

$$
\partial_{d} u(x)=\sum_{l=1}^{d-1}\left(\psi_{l}\right)_{x_{d}} * \partial_{l} g\left(x^{\prime}\right)
$$

with $\psi_{l}\left(x^{\prime}\right)=-x_{l} \varphi\left(x^{\prime}\right)$. Repeating this procedure, we get, for $|\alpha| \leq m-1$,

$$
\partial^{\alpha} u(x)=\sum_{\beta \in \mathbb{N}_{0}^{d-1}:|\beta|=|\alpha|}\left(\psi_{\alpha \beta}\right)_{x_{d}} * \partial^{\beta} g\left(x^{\prime}\right)
$$

with some $\psi_{\alpha \beta} \in C_{0}^{\infty}\left(\mathbb{R}^{d-1}\right)$, and

$$
\left\|\partial^{\alpha} u\left(\cdot, x_{d}\right)\right\|_{L_{p}\left(\mathbb{R}^{d-1}\right)} \leq C\|g\|_{W_{p}^{m-1}\left(\mathbb{R}^{d-1}\right)} .
$$

For $|\alpha|=m-1$ and $1 \leq j \leq d$ we have

$$
\partial_{j} \partial^{\alpha} u(x)=\sum_{l=1}^{d-1} \sum_{\beta \in \mathbb{N}_{0}^{d-1}:|\beta|=m-1} x_{d}^{-1}\left(\psi_{j l \alpha \beta}\right)_{x_{d}} * \partial^{\beta} g\left(x^{\prime}\right)
$$

with the functions

$$
\psi_{j l \alpha \beta}\left(x^{\prime}\right)= \begin{cases}\delta_{j l} \partial_{j} \psi_{\alpha \beta}\left(x^{\prime}\right) & \text { for } 1 \leq j \leq d-1 \\ -\partial_{l}\left(x_{l} \psi_{\alpha \beta}\left(x^{\prime}\right)\right) & \text { for } j=d\end{cases}
$$

where $\delta_{j l}$ is Kronecker's delta. Since $\int_{\mathbb{R}^{d-1}} \psi_{j l \alpha \beta}\left(x^{\prime}\right) d x^{\prime}=0$, we apply the result of Step 1 to get

$$
\left\|\partial_{j} \partial^{\alpha} u\right\|_{L_{p}\left(\mathbb{R}^{d}\right)} \leq C \sum_{\beta \in \mathbb{N}_{0}^{d-1}:|\beta|=m-1}\left|\partial^{\beta} g\right|_{B_{p p}^{1-1 / p}\left(\mathbb{R}^{d-1}\right)}=C|g|_{B_{p p}^{m-1 / p}\left(\mathbb{R}^{d-1}\right)} .
$$


Step 3. Let $g \in B_{p p}^{m-1 / p}\left(\mathbb{R}^{d-1}\right)$. We take a function $\eta \in C_{0}^{\infty}(\mathbb{R})$ satisfying $\eta(t)=1$ for $|t| \leq 1$, and $\operatorname{set} f(x)=\eta\left(x_{d}\right) u(x)$, where $u$ is defined by (5.6). It easily follows from (5.7) and (5.8) that $f \in W_{p}^{m}\left(\mathbb{R}_{+}^{d}\right)$ with $\|f\|_{W_{p}^{m}\left(\mathbb{R}_{+}^{d}\right)} \leq C\|g\|_{B_{p p}^{m-1 / p}\left(\mathbb{R}^{d-1}\right)}$.

It remains to check that $\operatorname{Tr} f=g$. To this end, we consider the translation $\tau_{h} f(x)=f\left(x^{\prime}, x_{d}+h\right)$ for $h>0$. It is easy to see that $\tau_{h} f \rightarrow f$ in $W_{p}^{m}\left(\mathbb{R}_{+}\right)$ as $h \rightarrow 0$. Hence the boundedness of the trace operator yields $\operatorname{Tr} \tau_{h} f \rightarrow \operatorname{Tr} f$ in $B_{p p}^{m-1 / p}\left(\mathbb{R}^{d-1}\right)$. On the other hand, by definition of $f$ we see that $\operatorname{Tr} \tau_{h} f=\varphi_{h} * g$ for $0<h<1$, and hence $\operatorname{Tr} \tau_{h} f \rightarrow g$ in $L_{p}\left(\mathbb{R}^{d-1}\right)$ as $h \rightarrow 0$. Therefore $\operatorname{Tr} f=g$.

\section{Convergence theorem}

In this section we consider the convergence of the integral

$$
T_{\epsilon} f=\int_{\epsilon}^{\lambda} t^{z} K_{t} * f \frac{d t}{t}
$$

as $\epsilon \rightarrow 0$ in the $L_{p}$ norm for $f \in L_{p}(\Omega), K \in \mathcal{K}_{\Gamma}^{0}$ and $z \in \mathbb{C}$. If $\operatorname{Re} z>0$, Proposition 2.9 guarantees its convergence since

$$
\begin{aligned}
\int_{0}^{\lambda}\left\|t^{z} K_{t} * f\right\|_{L_{p}} \frac{d t}{t} & \leq\|K\|_{L_{1}}\|f\|_{L_{p}} \int_{0}^{\lambda} t^{\operatorname{Re} z-1} d t \\
& =\frac{\|K\|_{L_{1}}\|f\|_{L_{p}}}{\operatorname{Re} z}<\infty
\end{aligned}
$$

If $\operatorname{Re} z=0$, the matter of convergence is subtle. When $z=0$, and $K$ is written as (2.6) with a function $\varphi \in \mathcal{K}_{\Gamma}^{0}$, we know that $T_{\epsilon} f$ converges to $\left(\int_{\mathbb{R}^{d}} \varphi(x) d x\right) f-\varphi_{\lambda} * f$ in $L_{p}(\Omega)$ since $T_{\epsilon} f=\varphi_{\epsilon} * f-\varphi_{\lambda} * f$ by $(2.7)$, although this case is beyond the scope of Proposition 2.9. Theorem 6.1 below enables us to assert that $T_{\epsilon} f$ converges even when $T_{\epsilon} f$ is not necessarily written in the form of $\varphi_{\epsilon} * f-\varphi_{\lambda} * f$, if the additional condition $K \in \mathcal{K}_{\Gamma}^{1}$ is assumed. It is also important to evaluate $T_{\epsilon} f$ with a bound independent of $z$, which can not be obtained from estimate (6.2) depending on $z$.

Theorem 6.1. Let $1<p<\infty, b \geq 0$, and $K \in \mathcal{K}_{\Gamma}^{1}$. For $f \in L_{p}(\Omega), 0<\epsilon<\lambda<\infty$ and $z \in \mathbb{C}$ with $0 \leq \operatorname{Re} z \leq b$ we consider $T_{\epsilon} f$ defined by (6.1). Then we have

$$
\left\|T_{\epsilon} f\right\|_{L_{p}(\Omega)} \leq C\|f\|_{L_{p}(\Omega)}, \quad \text { for } f \in L_{p}(\Omega)
$$

with $C=C\left(p, d, K, \lambda^{b}\right)$. Furthermore, $T_{\epsilon} f$ converges uniformly with respect to $z$ in $L_{p}(\Omega)$ as $\epsilon \rightarrow 0$. The limit function $T f=\lim _{\epsilon \rightarrow 0} T_{\epsilon} f$ satisfies, with the same $C$ as in (6.3),

$$
\|T f\|_{L_{p}(\Omega)} \leq C\|f\|_{L_{p}(\Omega)} .
$$

Proof. Extending $f \in L_{p}(\Omega)$ to $\mathbb{R}^{d}$ by zero, we may assume $\Omega=\mathbb{R}^{d}$. First we shall show (6.3). For the convergence of $T_{\epsilon} f$, it is crucial that the constant $C$ in (6.3) is independent of $\epsilon$. We need to take an approach different from the calculations in (6.2). 
Let $p=2$. We denote by $\mathcal{F} f$ the Fourier transform of $f$ :

$$
\mathcal{F} f(\xi)=\int_{\mathbb{R}^{d}} e^{-2 \pi i x \xi} f(x) d x .
$$

Then

$$
\mathcal{F}\left[T_{\epsilon} f\right](\xi)=\int_{\epsilon}^{\lambda} t^{z} \mathcal{F} K(t \xi) \mathcal{F} f(\xi) \frac{d t}{t}=\kappa(\xi) \mathcal{F} f(\xi)
$$

with $\kappa(\xi)=\int_{\epsilon}^{\lambda} t^{z} \mathcal{F} K(t \xi) t^{-1} d t$. By assumption we may write

$$
K=\sum_{|\alpha|=1} \partial^{\alpha} K_{\alpha}
$$

with some $K_{\alpha} \in C_{0}^{\infty}\left(\mathbb{R}^{d}\right)$. Since $\mathcal{F} K(\xi)=\sum_{|\alpha|=1}(2 \pi i \xi)^{\alpha} \mathcal{F} K_{\alpha}(\xi)$ and $\mathcal{F} K_{\alpha} \in \mathcal{S}\left(\mathbb{R}^{d}\right)$, we have $|\mathcal{F} K(\xi)| \leq C_{1} \min \left\{|\xi|,|\xi|^{-1}\right\}$. Let $\Lambda=\max \left\{\lambda^{b}, 1\right\}$, and note that $t^{\operatorname{Re} z} \leq$ $\lambda^{\operatorname{Re} z} \leq \Lambda$ for $0<t \leq \lambda$ and $0 \leq \operatorname{Re} z \leq b$. Changing the variables $t \rightarrow t|\xi|^{-1}$, we have

$$
\begin{aligned}
|\kappa(\xi)| & \leq \int_{\epsilon}^{\lambda} \Lambda C_{1} \min \left\{t|\xi|, t^{-1}|\xi|^{-1}\right\} \frac{d t}{t} \\
& \leq C_{1} \Lambda \int_{0}^{\infty} \min \left\{t, t^{-1}\right\} \frac{d t}{t} \\
& =2 C_{1} \Lambda .
\end{aligned}
$$

Plancherel's theorem gives

$$
\left\|T_{\epsilon} f\right\|_{L_{2}}^{2}=\|\kappa \mathcal{F} f\|_{L_{2}}^{2} \leq\left(2 C_{1} \Lambda\|\mathcal{F} f\|_{L_{2}}\right)^{2}=\left(2 C_{1} \Lambda\|f\|_{L_{2}}\right)^{2} .
$$

Thus we obtain (6.3) for $p=2$. In order to show (6.3) for $p \neq 2$ we require the Calderón-Zygmund theory of singular integral operators. We postpone the proof of (6.3) for $p \neq 2$ to the Appendix.

Assuming that (6.3) is true, we proceed to complete the proof. Let $f \in C_{0}^{\infty}\left(\mathbb{R}^{d}\right)$. For $0<\epsilon<\delta<\lambda$,

$$
T_{\delta} f-T_{\epsilon} f=\int_{\epsilon}^{\delta} \sum_{|\alpha|=1} t^{z}\left(K_{\alpha}\right)_{t} * f^{(\alpha)} d t,
$$

which gives $\left\|T_{\delta} f-T_{\epsilon} f\right\|_{L_{p}} \leq C \Lambda(\delta-\epsilon)\|\nabla f\|_{L_{p}}$. Hence $T_{\epsilon} f$ converges in $L_{p}\left(\mathbb{R}^{d}\right)$ as $\epsilon \rightarrow 0$.

Finally, let $f \in L_{p}\left(\mathbb{R}^{d}\right)$. Writing

$$
T_{\delta} f-T_{\epsilon} f=T_{\delta}(f-g)-T_{\epsilon}(f-g)+\left(T_{\delta} g-T_{\epsilon} g\right)
$$

with any $g \in C_{0}^{\infty}\left(\mathbb{R}^{d}\right)$, and using (6.3) and the above inequality for functions in $C_{0}^{\infty}\left(\mathbb{R}^{d}\right)$, we have

$$
\left\|T_{\delta} f-T_{\epsilon} f\right\|_{L_{p}} \leq 2 C\|f-g\|_{L_{p}}+C \Lambda(\delta-\epsilon)\|\nabla g\|_{L_{p}} .
$$

Since $C_{0}^{\infty}\left(\mathbb{R}^{d}\right)$ is dense in $L_{p}\left(\mathbb{R}^{d}\right),\left\{T_{\epsilon} f\right\}_{0<\epsilon<\lambda}$ satisfies Cauchy's convergence criterion. Therefore $T_{\epsilon} f$ converges in $L_{p}\left(\mathbb{R}^{d}\right)$. Letting $\epsilon \rightarrow 0$ in (6.3), we get $\|T f\|_{L_{p}} \leq$ $C\|f\|_{L_{p}}$. Thus we complete the proof. 
Corollary 6.2. Let $1<p<\infty, z \in \mathbb{C}$ with $\operatorname{Re} z>0$ and $K \in \mathcal{K}_{\Gamma}^{1}$. For $f \in L_{p}(\Omega)$ set

$$
F=\int_{0}^{\lambda} t^{z} K_{t} * f \frac{d t}{t}
$$

where the integral converges in $L_{p}(\Omega)$ by Proposition 2.9. If a multi-index $\alpha$ satisfies $0<|\alpha| \leq \operatorname{Re} z$, then $\partial^{\alpha} F \in L_{p}(\Omega)$ and

$$
\partial^{\alpha} F=\int_{0}^{\lambda} \partial^{\alpha}\left(t^{z} K_{t} * f\right) \frac{d t}{t}=\int_{0}^{\lambda} t^{z-|\alpha|}\left(K^{(\alpha)}\right)_{t} * f \frac{d t}{t} .
$$

Proof. We note that

$$
\partial^{\alpha} \int_{\epsilon}^{\lambda} t^{z} K_{t} * f \frac{d t}{t}=\int_{\epsilon}^{\lambda} \partial^{\alpha}\left\{t^{z} K_{t} * f\right\} \frac{d t}{t}
$$

for $0<\epsilon<\lambda$. Since $\operatorname{Re}(z-|\alpha|) \geq 0$, the last integral in the corollary converges in $L_{p}(\Omega)$ by Theorem 6.1. So the corollary follows by Lemma 2.1 .

With Theorem 6.1 and Corollary 6.2 in hand, we can improve Corollary 2.10, the interpolation inequality, in terms of pure derivatives.

Theorem 6.3. Let $1<p<\infty$ and $m \in \mathbb{N}$. Suppose that $f \in L_{p}(\Omega)$ and $\partial_{j}^{m} f \in L_{p}(\Omega)$ for all $j=1, \ldots, d$. Then $f \in W_{p}^{m}(\Omega)$, and the inequality

$$
\left\|\nabla^{k} f\right\|_{L_{p}(\Omega)} \leq C\left(\|f\|_{L_{p}(\Omega)}+\sum_{j=1}^{d}\left\|\partial_{j}^{m} f\right\|_{L_{p}(\Omega)}\right)
$$

holds for $1 \leq k \leq m$ with $C=C(m, p, d, \Omega)$. In particular,

$$
\left\|\nabla^{m} f\right\|_{L_{p}(\Omega)} \leq C \sum_{j=1}^{d}\left\|\partial_{j}^{m} f\right\|_{L_{p}(\Omega)} .
$$

Proof. We write Muramatu's formula (2.11) as

$$
f=\int_{0}^{\lambda} \sum_{|\beta|=m d}\left(\partial^{\beta} K_{\beta}\right)_{t} * f \frac{d t}{t}+\varphi_{\lambda} * f
$$

with $K_{\beta} \in \mathcal{K}_{\Gamma}^{1}$. For each $\beta$ with $|\beta|=m d$ there exists $j$ such that $\beta \geq m e_{j}$, where $e_{j}$ is the multi-index of length 1 whose $j$ th component is 1 ; otherwise we would have $|\beta| \leq(m-1) d<m d$, a contradiction. For $\beta$ with $|\beta|=m d$ and $\beta \geq m e_{j}$ we have

$$
\left(\partial^{\beta} K_{\beta}\right)_{t} * f=t^{m}\left(\partial^{\beta-m e_{j}} K_{\beta}\right)_{t} * f^{\left(m e_{j}\right)},
$$

so $f$ is written in the form

$$
f=\int_{0}^{\lambda} \sum_{j=1}^{d} t^{m}\left(K_{j}\right)_{t} * \partial_{j}^{m} f \frac{d t}{t}+\varphi_{\lambda} * f
$$


with some $K_{j} \in \mathcal{K}_{\Gamma}^{1}$. Applying Corollary 6.2, we find that $f \in W_{p}^{m}(\Omega)$ and

$$
\partial^{\alpha} f=\int_{0}^{\lambda} \sum_{j=1}^{d} t^{m-|\alpha|}\left(K_{j}^{(\alpha)}\right)_{t} * \partial_{j}^{m} f \frac{d t}{t}+\lambda^{-|\alpha|}\left(\varphi^{(\alpha)}\right)_{\lambda} * f
$$

for $|\alpha| \leq m$. Using Proposition 2.9 for $|\alpha|<m$ and Theorem 6.1 with $b=0$ for $|\alpha|=m$, we get

$$
\left\|\nabla^{k} f\right\|_{L_{p}} \leq C \lambda^{m-k} \sum_{j=1}^{d}\left\|\partial_{j}^{m} f\right\|_{L_{p}}+C \lambda^{-k}\|f\|_{L_{p}}
$$

for $k \leq m$. Estimate (6.4) follows by setting $\lambda=1$, and (6.5) follows by letting $\lambda \rightarrow \infty$ for $k=m$.

\section{Extension to the whole space}

In this section we construct an extension operator from $W_{p}^{m}(\Omega)$ to $W_{p}^{m}\left(\mathbb{R}^{d}\right)$.

Let $R_{\Omega}$ be the restriction, that is,

$$
R_{\Omega} f(x)=f(x), \quad x \in \Omega
$$

for functions $f$ defined on $\mathbb{R}^{d}$. Obviously, $R_{\Omega}: W_{p}^{m}\left(\mathbb{R}^{d}\right) \rightarrow W_{p}^{m}(\Omega)$ is a bounded linear operator, since $\partial^{\alpha}\left(R_{\Omega} f\right)=R_{\Omega}\left(\partial^{\alpha} f\right)$ and $\left\|\partial^{\alpha}\left(R_{\Omega} f\right)\right\|_{L_{p}(\Omega)} \leq\left\|\partial^{\alpha} f\right\|_{L_{p}\left(\mathbb{R}^{d}\right)}$ for $|\alpha| \leq m$.

Theorem 7.1. Let $1<p<\infty$ and $m \in \mathbb{N}_{0}$. Then there exists a bounded linear operator

$$
E_{\Omega}: W_{p}^{m}(\Omega) \rightarrow W_{p}^{m}\left(\mathbb{R}^{d}\right)
$$

such that $R_{\Omega} E_{\Omega} f=f$ for all $f \in W_{p}^{m}(\Omega)$, and that

$$
\left\|E_{\Omega} f\right\|_{W_{p}^{m}\left(\mathbb{R}^{d}\right)} \leq C\|f\|_{W_{p}^{m}(\Omega)}
$$

with $C=C(m, p, d, \Omega)$.

Proof. We use Muramatu's formula (2.14) with $\lambda=1$ :

$$
f=\int_{0}^{1} \sum_{|\beta|=m} t^{m}\left(K_{\beta}\right)_{t} * f^{(\beta)} \frac{d t}{t}+\varphi * f .
$$

Recall that $E_{0}$ is the zero-extension to $\mathbb{R}^{d}$ given by $(2.9)$, and define $E_{\Omega}$ by

$$
E_{\Omega} f=\int_{0}^{1} \sum_{|\beta|=m} t^{m}\left(K_{\beta}\right)_{t} * E_{0} f^{(\beta)} \frac{d t}{t}+\varphi * E_{0} f, \quad \text { for } f \in W_{p}^{m}(\Omega) .
$$

Let $f_{\epsilon}$ and $F_{\epsilon}$ be defined by the right-hand sides of (7.1) and (7.2), respectively, with $\int_{0}^{1}$ replaced by $\int_{\epsilon}^{1}$. Since $g \in L_{p}(\Omega)$ satisfies $R_{\Omega}\left(K * E_{0} g\right)=K * g$ for $K=K_{\beta}$ or $K=\varphi$, we have $R_{\Omega} F_{\epsilon}=f_{\epsilon}$. Since $f_{\epsilon} \rightarrow f$ in $L_{p}(\Omega)$, and $F_{\epsilon} \rightarrow E_{\Omega} f$ in $L_{p}\left(\mathbb{R}^{d}\right)$ as $\epsilon \rightarrow 0$, the boundedness of $R_{\Omega}$ gives $R_{\Omega} E_{\Omega} f=f$. 
By Corollary 6.2 it follows that $E_{\Omega} f \in W_{p}^{m}\left(\mathbb{R}^{d}\right)$ and

$$
\partial^{\alpha} E_{\Omega} f=\int_{0}^{1} \sum_{|\beta|=m} t^{m-|\alpha|}\left(K_{\beta}^{(\alpha)}\right)_{t} * E_{0} f^{(\beta)} \frac{d t}{t}+\varphi^{(\alpha)} * E_{0} f .
$$

Using Proposition 2.9 for $|\alpha|<m$ and Theorem 6.1 for $|\alpha|=m$, we have

$$
\begin{aligned}
\left\|\partial^{\alpha} E_{\Omega} f\right\|_{L_{p}\left(\mathbb{R}^{d}\right)} & \leq C \sum_{|\beta|=m}\left\|E_{0} f^{(\beta)}\right\|_{L_{p}\left(\mathbb{R}^{d}\right)}+C\left\|E_{0} f\right\|_{L_{p}\left(\mathbb{R}^{d}\right)} \\
& \leq C\left(\left\|\nabla^{m} f\right\|_{L_{p}(\Omega)}+\|f\|_{L_{p}(\Omega)}\right),
\end{aligned}
$$

which gives the boundedness of $E_{\Omega}$.

\section{Complex interpolation of Sobolev spaces}

In this section we show that the complex interpolation of two Sobolev spaces is a Sobolev space. To this end, we quickly review the method of complex interpolation. See $[3,35]$ for the details.

Let $X$ and $Y$ be Banach spaces with $Y \subset X$, and suppose that the embed$\operatorname{ding} Y \rightarrow X$ is continuous. Let $\mathcal{H}(X, Y)$ be the space of all $X$-valued functions $F$ satisfying the following conditions:

(i) $F$ is analytic in the strip

$$
S=\{z \in \mathbb{C}: 0<\operatorname{Re} z<1\} .
$$

(ii) $F$ is bounded and continuous on $\bar{S}$, the closure of $S$.

(iii) The map $s \mapsto F(1+i s)$ is a continuous function from $\mathbb{R}$ to $Y$.

We equip $\mathcal{H}(X, Y)$ with the norm

$$
\|F\|_{\mathcal{H}(X, Y)}=\max \left\{\sup _{s \in \mathbb{R}}\|F(i s)\|_{X}, \sup _{s \in \mathbb{R}}\|F(1+i s)\|_{Y}\right\} .
$$

Definition 8.1. For $0<\theta<1$ we define the complex interpolation space $[X, Y]_{\theta}$ by

$$
[X, Y]_{\theta}=\{F(\theta): F \in \mathcal{H}(X, Y)\}
$$

and equip $[X, Y]_{\theta}$ with the norm

$$
\|f\|_{[X, Y]_{\theta}}=\inf \left\{\|F\|_{\mathcal{H}(X, Y)}: F \in \mathcal{H}(X, Y) \text { and } F(\theta)=f\right\} .
$$

The space $[X, Y]_{\theta}$ is a Banach space.

Theorem 8.2. Let $1<p<\infty, n \in \mathbb{N}_{0}, m, k \in \mathbb{N}$ and $n<k<m$. Define $\theta \in(0,1)$ so that $k=(1-\theta) n+\theta m$. Then

$$
\left[W_{p}^{n}(\Omega), W_{p}^{m}(\Omega)\right]_{\theta}=W_{p}^{k}(\Omega)
$$

with equivalent norms. 
Proof. Step 1. First we shall show that $W_{p}^{k}(\Omega) \subset\left[W_{p}^{n}(\Omega), W_{p}^{m}(\Omega)\right]_{\theta}$.

Let $f \in W_{p}^{k}(\Omega)$. Taking into account that $f$ is written as

$$
f=\int_{0}^{1} \sum_{|\beta|=k} t^{k}\left(K_{\beta}\right)_{t} * f^{(\beta)} \frac{d t}{t}+\varphi * f
$$

with $K_{\beta} \in \mathcal{K}_{\Gamma}^{1}$ by Muramatu's formula (2.14), we set

$$
F(z)=\int_{0}^{1} \sum_{|\beta|=k} t^{(1-z) n+z m}\left(K_{\beta}\right)_{t} * f^{(\beta)} \frac{d t}{t}+\varphi * f, \quad \text { for } z \in \bar{S} .
$$

Obviously, $F(\theta)=f$. Also, $\varphi * f$ is a constant term belonging to $W_{p}^{m}(\Omega)$. In order to show $F \in \mathcal{H}\left(W_{p}^{n}(\Omega), W_{p}^{m}(\Omega)\right)$, we define $F_{\epsilon}(z)$ to be the right-hand side of the above equation with $\int_{0}^{1}$ replaced by $\int_{\epsilon}^{1}$ for $\epsilon>0$. By the continuity and analyticity of $z \mapsto t^{(1-z) n+z m}$, and by (6.3) it is easy to see that $F_{\epsilon} \in \mathcal{H}\left(W_{p}^{n}(\Omega), W_{p}^{m}(\Omega)\right)$ with $\left\|F_{\epsilon}\right\|_{W_{p}^{n}} \leq C\|f\|_{W_{p}^{k}}$ with $C$ independent of $\epsilon$. Since $n \leq \operatorname{Re}\{n(1-z)+m z\} \leq m$, it follows by Theorem 6.1 and Corollary 6.2 that $F_{\epsilon}(z)$ converges to $F(z)$ in $W_{p}^{n}(\Omega)$ as $\epsilon \rightarrow 0$ uniformly with respect to $z \in \bar{S}$. Also, $F_{\epsilon}(z)$ converges to $F(z)$ in $W_{p}^{m}(\Omega)$ uniformly on the line $\operatorname{Re} z=1$. Therefore we conclude that $F \in \mathcal{H}\left(W_{p}^{n}(\Omega), W_{p}^{m}(\Omega)\right)$ with $\|f\|_{\left[W_{p}^{n}(\Omega), W_{p}^{m}(\Omega)\right]_{\theta}} \leq C\|f\|_{W_{p}^{k}(\Omega)}$.

Step 2. Next we shall show that $\left[W_{p}^{n}(\Omega), W_{p}^{m}(\Omega)\right]_{\theta} \subset W_{p}^{k}(\Omega)$.

Let $f \in\left[W_{p}^{n}(\Omega), W_{p}^{m}(\Omega)\right]_{\theta}$, and take $F \in \mathcal{H}\left(W_{p}^{n}(\Omega), W_{p}^{m}(\Omega)\right)$ so that $F(\theta)=f$. Our goal is to show the inequality

$$
\left|\int_{\Omega} f(x) \partial^{\alpha} g(x) d x\right| \leq C\|F\|_{\mathcal{H}\left(W_{p}^{n}(\Omega), W_{p}^{m}(\Omega)\right)}\|g\|_{L_{p^{\prime}}(\Omega)}
$$

for all $g \in C_{0}^{\infty}(\Omega)$ and $|\alpha| \leq k$ with $1 / p+1 / p^{\prime}=1$.

To this end, we view $g \in C_{0}^{\infty}(\Omega)$ as a function belonging to $C_{0}^{\infty}\left(\mathbb{R}^{d}\right) \subset L_{p^{\prime}}\left(\mathbb{R}^{d}\right)$, and use Muramatu's formula (2.11) for $\partial^{\alpha} g$, taking $-\Gamma$ as the cone associated with the whole space $\mathbb{R}^{d}$; recall that $\Gamma$ is the cone associated with $\Omega$. Then we have

$$
\partial^{\alpha} g=\int_{0}^{1} H_{t} * g^{(\alpha)} \frac{d t}{t}+\psi * g^{(\alpha)}=\int_{0}^{1} t^{-|\alpha|}\left(H^{(\alpha)}\right)_{t} * g \frac{d t}{t}+\psi^{(\alpha)} * g
$$

with $H \in \mathcal{K}_{-\Gamma}^{2 m+1}$ and $\psi \in \mathcal{K}_{-\Gamma}^{0}$. Taking this formula into account, we define the function $z \mapsto G(z) \in L_{p^{\prime}}\left(\mathbb{R}^{d}\right)$ on $\bar{S}$ by

$$
G(z)=\int_{0}^{1} t^{k-|\alpha|-(1-z) n-z m}\left(H^{(\alpha)}\right)_{t} * g \frac{d t}{t}+\psi^{(\alpha)} * g .
$$

Obviously, $G(\theta)=\partial^{\alpha} g$. Since $G(z)$ is written as

$$
G(z)=\int_{0}^{1} t^{k-|\alpha|-(1-z) n-z m+m+1} \sum_{|\beta|=m+1}\left(H_{\beta}\right)_{t} * g^{(\beta)} \frac{d t}{t}+\psi^{(\alpha)} * g
$$

with some $H_{\beta} \in \mathcal{K}_{-\Gamma}^{1}$, and since $\operatorname{Re}(k-|\alpha|-(1-z) n-z m+m+1) \geq 1$ for $z \in \bar{S}$, the same argument as for $F_{\epsilon}(z)$ in Step 1 shows that $G(z)$ is an $L_{p^{\prime}}\left(\mathbb{R}^{d}\right)$-valued function that is analytic in $S$, and bounded and continuous on $\bar{S}$. 
By Corollary 6.2 the derivative $\partial^{\gamma} G(z)$ with $|\gamma|=1$ is equal to the right-hand side of $(8.3)$ with $\left(H_{\beta}\right)_{t} * g^{(\beta)}$ and $\psi^{(\alpha)} * g$ replaced by $\partial^{\gamma}\left(H_{\beta}\right)_{t} * g^{(\beta)}=\left(H_{\beta}\right)_{t} * g^{(\beta+\gamma)}$ and $\psi^{(\alpha)} * g^{(\gamma)}$, respectively. Repeating this procedure, we get the similar expression of $\partial^{\gamma} G(z)$ for every multi-index $\gamma$. Hence $G(z) \in W_{p^{\prime}}^{l}\left(\mathbb{R}^{d}\right)$ for all $l \in \mathbb{N}$. By the embedding theorem (Theorem 4.2) we know $G(z) \in C^{\infty}\left(\mathbb{R}^{d}\right)$. Furthermore, we find $G(z) \in C_{0}^{\infty}(\Omega)$, since $\operatorname{supp} H_{\beta}$ and $\operatorname{supp} \psi$ are subsets of $-(-\Gamma)_{0}=\Gamma_{0}$ by Definition 2.4 , and therefore

$$
\bigcup_{0 \leq t \leq 1} \operatorname{supp}\left(H_{\beta}\right)_{t} * g^{(\beta)} \text { and } \operatorname{supp} \psi^{(\alpha)} * g
$$

are compact and contained in $\operatorname{supp} g+\Gamma_{0} \subset \Omega$.

Now we set $I(z)=\int_{\Omega} F(z) G(z) d x$ and evaluate $I(z)$, which is analytic in $S$, and bounded and continuous on $\bar{S}$ by the above properties of $F(z)$ and $G(z)$. Observe that $H^{(\alpha)}$ is written as $H^{(\alpha)}=\sum_{|\beta|=m} \partial^{\beta} M_{\beta}$ with $M_{\beta} \in \mathcal{K}_{-\Gamma}^{m+1}$, and that

$$
\left(H^{(\alpha)}\right)_{t} * g=t^{m} \sum_{|\beta|=m} \partial^{\beta}\left\{\left(M_{\beta}\right)_{t} * g\right\}=t^{m} \sum_{|\beta|=m}\left(M_{\beta}\right)_{t} * g^{(\beta)} .
$$

For $z=1+i s$ with $s \in \mathbb{R}$ we can rewrite (8.2) as

$$
G(1+i s)=\sum_{|\beta|=m} \partial^{\beta} \int_{0}^{1} t^{k-|\alpha|-i(m-n) s}\left(M_{\beta}\right)_{t} * g \frac{d t}{t}+\psi^{(\alpha)} * g,
$$

where we interchanged the order of $\partial^{\beta}$ and integration by Proposition 2.9 and Theorem 6.1. By integration by parts and Theorem 6.1 we have

$$
\begin{aligned}
|I(1+i s)| \leq & \sum_{|\beta|=m}\left\|\partial^{\beta} F(1+i s)\right\|_{L_{p}}\left\|\int_{0}^{1} t^{k-|\alpha|-i(m-n) s}\left(M_{\beta}\right)_{t} * g \frac{d t}{t}\right\|_{L_{p^{\prime}}} \\
& \quad+\|F(1+i s)\|_{L_{p}}\left\|\psi^{(\alpha)} * g\right\|_{L_{p^{\prime}}} \\
\leq C & \sup _{s \in \mathbb{R}}\|F(1+i s)\|_{W_{p}^{m}(\Omega)}\|g\|_{L_{p^{\prime}}}
\end{aligned}
$$

Similarly, $|I(i s)| \leq C \sup _{s \in \mathbb{R}}\|F(i s)\|_{W_{p}^{n}(\Omega)}\|g\|_{L_{p^{\prime}}}$. Since

$$
I(\theta)=\int_{\Omega} f(x) \partial^{\alpha} g(x) d x
$$

the maximum principle for analytic functions yields (8.1). From the duality between $L_{p}(\Omega)$ and $L_{p^{\prime}}(\Omega)$ (see [12, Theorem 6.15]), and the fact that $C_{0}^{\infty}(\Omega)$ is dense in $L_{p^{\prime}}(\Omega)$ it follows that $\partial^{\alpha} f \in L_{p}(\Omega)$ for $|\alpha| \leq k$, and that $\|f\|_{W_{p}^{k}(\Omega)} \leq$ $C\|F\|_{\mathcal{H}\left(W_{p}^{n}(\Omega), W_{p}^{m}(\Omega)\right)}$. Taking the infimum of the right-hand side, we conclude that $\left[W_{p}^{n}(\Omega), W_{p}^{m}(\Omega)\right]_{\theta} \subset W_{p}^{k}(\Omega)$ with $\|f\|_{W_{p}^{k}(\Omega)} \leq C\|f\|_{\left[W_{p}^{n}(\Omega), W_{p}^{m}(\Omega)\right]_{\theta}}$.

\section{Real interpolation of Sobolev spaces}

In this section we show that the real interpolation of two Sobolev spaces is a Besov space. To this end, we quickly review the method of real interpolation. See $[1,3,35]$ 
for the details. There are several ways to define real interpolation spaces, of which we adopt the $K$-method. Recall that $L_{q}^{*}\left(\mathbb{R}^{d}\right)$ and $L_{q}^{*}\left(\mathbb{R}_{+}\right)$are defined by (5.1).

Definition 9.1. Let $X$ and $Y$ be Banach spaces with $Y \subset X$, and suppose that $\|f\|_{X} \leq\|f\|_{Y}$. For $f \in X$ and $t>0$ we set

$$
\mathbb{K}(t, f)=\inf \left\{\|u\|_{X}+t\|v\|_{Y}: f=u+v, u \in X, v \in Y\right\} .
$$

For $0<\theta<1$ and $1 \leq q \leq \infty$ we define the real interpolation space $(X, Y)_{\theta, q}$ by

$$
(X, Y)_{\theta, q}=\left\{f \in X: t^{-\theta} \mathbb{K}(t, f) \in L_{q}^{*}\left(\mathbb{R}_{+}\right)\right\}
$$

and equip it with the norm

$$
\|f\|_{(X, Y)_{\theta, q}}=\left\|t^{-\theta} \mathbb{K}(t, f)\right\|_{L_{q}^{*}\left(\mathbb{R}_{+}\right)} .
$$

The real interpolation space $(X, Y)_{\theta, q}$ is a Banach space. If we write $f \in X$ as $f=u+v$ with $u \in X$ and $v \in Y$, then $\|f\|_{X} \leq\|u\|_{X}+t\|v\|_{Y}$ for $t \geq 1$ by the triangle inequality and the assumption $\|v\|_{X} \leq\|v\|_{Y}$. Taking the infimum, we get $\|f\|_{X} \leq \mathbb{K}(t, f)$ and hence

$$
\|f\|_{(X, Y)_{\theta, q}} \geq\left\|t^{-\theta} \mathbb{K}(t, f)\right\|_{L_{q}\left([1, \infty), t^{-1} d t\right)} \geq(\theta q)^{-1 / q}\|f\|_{X},
$$

where $(\theta q)^{-1 / q}$ should be replaced by 1 if $q=\infty$. Also, it follows from $\mathbb{K}(t, f) \leq$ $\min \left\{\|f\|_{X}, t\|f\|_{Y}\right\} \leq \min \{1, t\}\|f\|_{Y}$ that $Y \subset(X, Y)_{\theta, q}$ with $\|f\|_{(X, Y)_{\theta, q}} \leq C(\theta, q)$. $\|f\|_{Y}$ for $f \in Y$.

For $m \in \mathbb{N}$ and $h \in \mathbb{R}^{d}$ we set

$$
\Omega_{m, h}=\bigcap_{j=0}^{m}(\Omega-j h),
$$

and define the $m$-th order difference operator by

$$
\Delta_{h}^{m} f(x)= \begin{cases}\sum_{k=0}^{m}(-1)^{m-k}\left(\begin{array}{c}
m \\
k
\end{array}\right) f(x+k h) & \left(x \in \Omega_{m, h}\right), \\
0 & \text { (otherwise) }\end{cases}
$$

for a function $f$ on $\Omega$. We note that $\Delta_{h}^{m} f=\Delta_{h}\left(\Delta_{h}^{m-1} f\right)$ for $m \geq 2$. To state the theorem for the real interpolation of Sobolev spaces, we introduce the space $B_{p q}^{m, \sigma}(\Omega)$, which will turn out to coincide with the Besov space $B_{p q}^{\sigma}(\Omega)$ defined in Definition 5.1 .

Definition 9.2. Let $1<p<\infty, 1 \leq q \leq \infty, m \in \mathbb{N}$ and $0<\sigma<m$. Set

$$
|f|_{B_{p q}^{m, \sigma}(\Omega)}=\left\|\frac{\left\|\Delta_{h}^{m} f\right\|_{L_{p}\left(\Omega_{m, h}\right)}}{|h|^{\sigma}}\right\|_{L_{q}^{*}\left(\mathbb{R}^{d}\right)}, \quad \text { for } f \in L_{p}(\Omega) .
$$

The space $B_{p q}^{m, \sigma}(\Omega)$ is defined by

$$
B_{p q}^{m, \sigma}(\Omega)=\left\{f \in L_{p}(\Omega):|f|_{B_{p q}^{m, \sigma}(\Omega)}<\infty\right\},
$$

and equipped with the norm $\|f\|_{L_{p}(\Omega)}+|f|_{B_{p q}^{m, \sigma}(\Omega)}$. 
Theorem 9.3. Let $1<p<\infty, 1 \leq q \leq \infty, m \in \mathbb{N}, 0<\theta<1$ and $\sigma=m \theta$. Then

$$
\left(L_{p}(\Omega), W_{p}^{m}(\Omega)\right)_{\theta, q}=B_{p q}^{\sigma}(\Omega)=B_{p q}^{m, \sigma}(\Omega)
$$

with equivalent norms.

Remark 9.4. By Theorem 9.3 we know that in the definition of $B_{p q}^{\sigma}(\Omega)$ with $\sigma>0$ we may replace $\Delta_{h}$ or $\Delta_{h}^{2}$ by $\Delta_{h}^{m}$ with any integer $m \geq 2$. Indeed, $f \in B_{p q}^{\sigma}(\Omega)$ if and only if $f \in W_{p}^{[\sigma]_{-}}(\Omega)$ and $f^{(\alpha)} \in B_{p q}^{\{\sigma\}_{+}}(\Omega)=B_{p q}^{m,\{\sigma\}_{+}}(\Omega)$ for $|\alpha|=[\sigma]_{-}$, where $m$ is an integer satisfying $m>\{\sigma\}_{+}$.

For the proof we prepare several lemmas.

Lemma 9.5. For $m \in \mathbb{N}$ define the sequence $\left\{b_{j}\right\}_{j=0}^{m}$ so that the identity

$$
\prod_{k=1}^{m-1}\left(1-\frac{m-k}{k} t\right)=\sum_{j=0}^{m-1} b_{j} t^{j}
$$

holds as polynomials in $t$. Let $K \in C_{0}^{\infty}\left(\mathbb{R}^{d}\right)$ be written in the form

$$
K(x)=\sum_{|\alpha|=m-1} K_{\alpha}^{(\alpha)}(x)
$$

with $K_{\alpha} \in \mathcal{K}_{\Gamma}^{0}$ satisfying $\int_{\mathbb{R}^{d}} K_{\alpha}(x) d x=0$, let $\omega \in \mathcal{K}_{\Gamma}^{0}$ satisfy $\int_{\mathbb{R}^{d}} \omega(x) d x=1$, and set

$$
\begin{aligned}
W(x, y)= & (-1)^{m} m^{d} \\
& \times \sum_{|\alpha|=m-1} \sum_{j=0}^{m-1}\left(\begin{array}{c}
m-1 \\
j
\end{array}\right)^{-1} b_{j} \sum_{\beta: \beta \leq \alpha,|\beta|=j}\left(\begin{array}{c}
\alpha \\
\beta
\end{array}\right) K_{\alpha}^{(\alpha-\beta)}(x) \omega^{(\beta)}(y), \\
W_{t}(x, y)= & t^{-2 d} W(x / t, y / t), \quad \text { for } t>0 .
\end{aligned}
$$

Then

$$
K_{t} * f(x)=\int_{\mathbb{R}^{d}} d z \int_{\Omega_{m, z}} W_{t}(x-y, x-y-m z) \Delta_{z}^{m} f(y) d y, \quad \text { for } x \in \Omega .
$$

Proof. We write $U(t, x)$ for the right-hand side of (9.3), which is well defined since the set $\left\{(y, z) \in \mathbb{R}^{2 d}: z \in \mathbb{R}^{d}, y \in \Omega_{m, z}\right\}$ is open, and hence measurable. If we set

$$
U_{k}(t, x):=\iint_{\mathbb{R}^{2 d}} W_{t}(x-y, x-y-m z) E_{0} f(y+k z) d y d z
$$

for $k \in\{0,1, \ldots, m\}$, we have

$$
U(t, x)=\sum_{k=0}^{m}(-1)^{m-k}\left(\begin{array}{c}
m \\
k
\end{array}\right) U_{k}(t, x) .
$$

Indeed, $W_{t}(x-y, x-y-m z) \neq 0$ implies $y \in x+t \Gamma_{0} \subset x+\Gamma$ and $y+m z \in x+\Gamma$, and the convexity of $\Gamma$ gives $y+k z \in x+\Gamma \subset \Omega$ for all $k$ with $0 \leq k \leq m$, so we may restrict the domain of $y$-integration for $U_{k}(t, x)$ to $\Omega_{m, z}$. Thus it suffices to evaluate each $U_{k}(t, x)$ for $x \in \Omega$. 
Let $k=0$. The substitution $w=x-y-m z$ gives

$$
U_{0}(t, x)=m^{-d} \iint_{\mathbb{R}^{2 d}} W_{t}(x-y, w) E_{0} f(y) d y d w .
$$

In view of $b_{0}=1$ and $\int_{\mathbb{R}^{d}}\left(\omega^{(\beta)}\right)_{t}(w) d w=0$ for $|\beta|>0$, we have

$$
\begin{aligned}
m^{-d} \int_{\mathbb{R}^{d}} W_{t}(x-y, w) d w & =\int_{\mathbb{R}^{d}}(-1)^{m} \sum_{|\alpha|=m-1}\left(K_{\alpha}^{(\alpha)}\right)_{t}(x-y) \omega_{t}(w) d w \\
& =(-1)^{m} K_{t}(x-y),
\end{aligned}
$$

which gives

$$
U_{0}(t, x)=(-1)^{m} K_{t} * E_{0} f(x)=(-1)^{m} K_{t} * f(x) .
$$

Let $k=m$. The substitution $w=y+m z$ and $\int_{\mathbb{R}^{d}}\left(K_{\alpha}^{(\alpha-\beta)}\right)_{t}(x-y) d y=0$ give

$$
U_{m}(t, x)=m^{-d} \iint_{\mathbb{R}^{2 d}} W_{t}(x-y, x-w) E_{0} f(w) d y d w=0 .
$$

Let $1 \leq k \leq m-1$. The substitution $w=y+k z$ gives

$$
U_{k}(t, x)=k^{-d} \iint_{\mathbb{R}^{2 d}} W_{t}\left(x-y, x+\frac{(m-k) y-m w}{k}\right) E_{0} f(w) d y d w .
$$

Integration by parts gives

$$
\begin{aligned}
& \int_{\mathbb{R}^{d}}\left(K_{\alpha}^{(\alpha-\beta)}\right)_{t}(x-y)\left(\omega^{(\beta)}\right)_{t}\left(x+\frac{(m-k) y-m w}{k}\right) d y \\
& =\left(\frac{k}{m-k}\right)^{|\beta|} \int_{\mathbb{R}^{d}}\left(K_{\alpha}^{(\alpha)}\right)_{t}(x-y) \omega_{t}\left(x+\frac{(m-k) y-m w}{k}\right) d y \\
& =:\left(\frac{k}{m-k}\right)^{|\beta|} V_{\alpha}(t, x, w),
\end{aligned}
$$

where we write the last integral as $V_{\alpha}(t, x, w)$. We observe the relation

$$
\sum_{\beta: \beta \leq \alpha,|\beta|=j}\left(\begin{array}{c}
\alpha \\
\beta
\end{array}\right)=\left(\begin{array}{c}
|\alpha| \\
j
\end{array}\right)
$$

for $j \leq|\alpha|$, which follows by setting $x=(t, t, \ldots, t)$ and $y=(1,1, \ldots, 1)$ in the identity $(x+y)^{\alpha}=\sum_{\beta \leq \alpha}\left(\begin{array}{l}\alpha \\ \beta\end{array}\right) x^{\beta} y^{\alpha-\beta}$. By this relation we have

$$
\begin{aligned}
& (-1)^{-m} m^{-d} \int_{\mathbb{R}^{d}} W_{t}\left(x-y, x+\frac{(m-k) y-m w}{k}\right) d y \\
& =\sum_{|\alpha|=m-1} \sum_{j=0}^{m-1}\left(\begin{array}{c}
m-1 \\
j
\end{array}\right)^{-1} b_{j} \sum_{\beta \leq \alpha,|\beta|=j}\left(\begin{array}{c}
\alpha \\
\beta
\end{array}\right)\left(\frac{k}{m-k}\right)^{j} V_{\alpha}(t, x, w) \\
& =\sum_{|\alpha|=m-1}\left\{\sum_{j=0}^{m-1} b_{j}\left(\frac{k}{m-k}\right)^{j}\right\} V_{\alpha}(t, x, w)=0,
\end{aligned}
$$

where we used the fact that the sum in the braces is equal to 0 by (9.2). Thus we get $U_{k}(t, x)=0$ for $1 \leq k \leq m-1$. 
Combining the estimates for all $U_{k}(t, x)$, we obtain $(9.3)$.

Lemma 9.6. Let $1<p<\infty$ and $m \in \mathbb{N}$. Then

$$
\left\|\Delta_{h}^{m} f\right\|_{L_{p}\left(\Omega_{m, h}\right)} \leq C|h|^{m}\|f\|_{W_{p}^{m}(\Omega)}, \quad \text { for } f \in W_{p}^{m}(\Omega)
$$

with $C=C(m, p, d, \Omega)$.

Proof. First we consider the case $\Omega=\mathbb{R}^{d}$. Since $W_{p}^{m}\left(\mathbb{R}^{d}\right) \cap C^{\infty}\left(\mathbb{R}^{d}\right)$ is dense in $W_{p}^{m}\left(\mathbb{R}^{d}\right)$ by Theorem 2.11 , we may assume that $f \in W_{p}^{m}\left(\mathbb{R}^{d}\right) \cap C^{\infty}\left(\mathbb{R}^{d}\right)$. By Taylor's theorem

$$
\begin{aligned}
\Delta_{h}^{m} f(x)= & \sum_{k=0}^{m} \sum_{|\alpha|<m}(-1)^{m-k}\left(\begin{array}{c}
m \\
k
\end{array}\right) \frac{(k h)^{\alpha} f^{(\alpha)}(x)}{\alpha !} \\
& +\sum_{k=0}^{m} \sum_{|\alpha|=m}(-1)^{m-k}\left(\begin{array}{c}
m \\
k
\end{array}\right) \frac{m(k h)^{\alpha}}{\alpha !} \int_{0}^{1}(1-s)^{m-1} f^{(\alpha)}(x+s k h) d s \\
= & : J_{0}+J_{1} .
\end{aligned}
$$

To estimate $J_{0}$ we consider the identity $(t-1)^{m}=\sum_{k=0}^{m}(-1)^{m-k}\left(\begin{array}{c}m \\ k\end{array}\right) t^{k}$ as polynomials in $t$, and apply $\{t(d / d t)\}^{l}$ with $0 \leq l<m$ to both sides. Then there exists a polynomial $P_{l}(t)$ such that

$$
P_{l}(t)(t-1)^{m-l}=\sum_{k=0}^{m}(-1)^{m-k}\left(\begin{array}{c}
m \\
k
\end{array}\right) k^{l} t^{k} .
$$

Putting $t=1$ gives $\sum_{k=0}^{m}(-1)^{m-k}\left(\begin{array}{c}m \\ k\end{array}\right) k^{l}=0$ and hence $J_{0}=0$. Applying Minkowski's inequality to $J_{1}$, we get (9.4) with $\|f\|_{W_{p}^{m}(\Omega)}$ replaced by $\left\|\nabla^{m} f\right\|_{L_{p}(\Omega)}$ for $\Omega=\mathbb{R}^{d}$.

Next we consider the case $\Omega \neq \mathbb{R}^{d}$. Let $E_{\Omega}$ be the extension operator in Theorem 7.1. By the result for $\mathbb{R}^{d}$ we have

$$
\left\|\Delta_{h}^{m} f\right\|_{L_{p}\left(\Omega_{m, h}\right)} \leq\left\|\Delta_{h}^{m} E_{\Omega} f\right\|_{L_{p}\left(\mathbb{R}^{d}\right)} \leq C|h|^{m}\left\|\nabla^{m} E_{\Omega} f\right\|_{L_{p}\left(\mathbb{R}^{d}\right)},
$$

which yields (9.4).

Lemma 9.7. Let $1<p<\infty, 1 \leq q \leq \infty$ and $0<\sigma \leq 1$. Then $W_{p}^{2}(\Omega) \subset B_{p q}^{\sigma}(\Omega)$ with $\|f\|_{B_{p q}^{\sigma}(\Omega)} \leq C(\sigma, p, q, d, \Omega)\|f\|_{W_{p}^{2}(\Omega)}$ for $f \in W_{p}^{2}(\Omega)$.

Proof. Let $f \in W_{p}^{2}(\Omega)$. It is easy to see that $\left\|\Delta_{h} f\right\|_{L_{p}\left(\Omega_{1, h}\right)} \leq 2\|f\|_{L_{p}(\Omega)}$. This combined with Lemma 9.6 gives

$$
\left\|\Delta_{h} f\right\|_{L_{p}\left(\Omega_{1, h}\right)} \leq C \min \{1,|h|\}\|f\|_{W_{p}^{1}(\Omega)} .
$$

If $0<\sigma<1$, then $|h|^{-\sigma} \min \{1,|h|\} \in L_{q}^{*}\left(\mathbb{R}^{d}\right)$ gives $f \in B_{p q}^{\sigma}(\Omega)$. If $\sigma=1$, the same argument yields the above inequality with $\Delta_{h} f$ and $\min \{1,|h|\}$ replaced by $\Delta_{h}^{2} f$ and $\min \left\{1,|h|^{2}\right\}$, respectively; hence we get $f \in B_{p q}^{1}(\Omega)$. 
Lemma 9.8. Let $1<p<\infty, 1 \leq q \leq \infty, m \in \mathbb{N}, 0<\sigma<m$ and $K \in \mathcal{K}_{\Gamma}^{m}$. If $f \in B_{p q}^{m, \sigma}(\Omega)$, then there exists $G_{i} \in L_{q}^{*}\left(\mathbb{R}_{+}\right)$with $i=0,1,2$ such that

$$
\begin{aligned}
\left\|K_{t} * f\right\|_{L_{p}(\Omega)} & \leq t^{\sigma} G_{0}(t), \\
\left\|\Delta_{h}^{i}\left(K_{t} * f\right)\right\|_{L_{p}\left(\Omega_{i, h}\right)} & \leq t^{\sigma} \min \left\{1,(|h| / t)^{i}\right\} G_{i}(t) \quad \text { for } i=1,2, \\
\left\|G_{i}\right\|_{L_{q}^{*}\left(\mathbb{R}_{+}\right)} & \leq C(m, \sigma, p, q, d, \Omega)|f|_{B_{p q}^{m, \sigma}(\Omega)} \quad \text { for } i=0,1,2 .
\end{aligned}
$$

Proof. We use Lemma 9.5 to write $K_{t} * f$ as (9.3). Since $W_{t}(x-y, x-y-m z) \neq 0$ implies $|x-y|<t,|x-y-m z|<t$ and hence $|m z|<2 t$, we have

$$
\left|W_{t}(x-y, x-y-m z)\right| \leq t^{-2 d}\|W\|_{L_{\infty}} \chi\left(\frac{|x-y|}{t}\right) \chi\left(\frac{m|z|}{2 t}\right),
$$

where $\chi$ is the characteristic function of $[0,1]$. Applying Minkowski's inequality and Young's inequality to (9.3), we have

$$
\left\|K_{t} * f\right\|_{L_{p}(\Omega)} \leq C_{0} \int_{\mathbb{R}^{d}} t^{-d} \chi\left(\frac{m|z|}{2 t}\right)\left\|\Delta_{z}^{m} f\right\|_{L_{p}\left(\Omega_{m, z}\right)} d z
$$

with some constant $C_{0}$. If we set

$$
G_{0}(t)=C_{0} \int_{\mathbb{R}^{d}}\left(\frac{|z|}{t}\right)^{\sigma+d} \chi\left(\frac{m|z|}{2 t}\right) \frac{\left\|\Delta_{z}^{m} f\right\|_{L_{p}\left(\Omega_{m, z}\right)}}{|z|^{\sigma}} \frac{d z}{|z|^{d}},
$$

then (9.5) holds. Since $\int_{0}^{\infty} t^{\sigma+d} \chi(m t / 2) t^{-1} d t<\infty$, the map

$$
|z|^{-\sigma}\left\|\Delta_{z}^{m} f\right\|_{L_{p}\left(\Omega_{m, z}\right)} \mapsto G_{0}(t)
$$

is a bounded operator from $L_{q}^{*}\left(\mathbb{R}^{d}\right)$ to $L_{q}^{*}\left(\mathbb{R}_{+}\right)$by Lemma 5.3. Therefore we obtain (9.7) for $i=0$.

As for (9.6) and (9.7) with $i=1$, we consider, instead of $W_{t}(x-y, x-y-m z)$, the difference

$$
J:=W_{t}(x+h-y, x+h-y-m z)-W_{t}(x-y, x-y-m z)
$$

for $x \in \Omega_{1, h}$. Taking into account the support of $J$, and using Taylor's theorem, we have

$$
|J| \leq\|J\|_{L_{\infty}}\left\{\chi\left(\frac{|x+h-y|}{t}\right)+\chi\left(\frac{|x-y|}{t}\right)\right\} \chi\left(\frac{m|z|}{2 t}\right)
$$

with $\|J\|_{L_{\infty}} \leq \min \left\{2\|W\|_{L_{\infty}},(|h| / t)\|\nabla W\|_{L_{\infty}}\right\} t^{-2 d}$. Applying $\Delta_{h}$ to (9.3), and using Minkowski's inequality with Young's inequality, we get

$$
\left\|\Delta_{h}\left(K_{t} * f\right)\right\|_{L_{p}\left(\Omega_{1, h}\right)} \leq C_{1} \min \{1,|h| / t\} \int_{\mathbb{R}^{d}} t^{-d} \chi\left(\frac{m|z|}{2 t}\right)\left\|\Delta_{z}^{m} f\right\|_{L_{p}\left(\Omega_{m, z}\right)} d z
$$

with some constant $C_{1}$. In the same way as for the case $i=0$, we obtain (9.6) and (9.7) for $i=1$.

The case $i=2$ can be handled in a similar way.

Proof of Theorem 9.3. Step 1. We shall show that

$$
\left(L_{p}(\Omega), W_{p}^{m}(\Omega)\right)_{\theta, q} \subset B_{p q}^{m, \sigma}(\Omega) .
$$


Let $f \in\left(L_{p}(\Omega), W_{p}^{m}(\Omega)\right)_{\theta, q}$. By definition $t^{-\theta} \mathbb{K}(t, f) \in L_{q}^{*}\left(\mathbb{R}_{+}\right)$, and for each $h \in \mathbb{R}^{d}$ there exist $u \in L_{p}(\Omega)$ and $v \in W_{p}^{m}(\Omega)$ such that $f=u+v$ and

$$
\|u\|_{L_{p}(\Omega)}+|h|^{m}\|v\|_{W_{p}^{m}(\Omega)} \leq 2 \mathbb{K}\left(|h|^{m}, f\right) .
$$

By Lemma 9.6 we have

$$
\begin{aligned}
\left\|\Delta_{h}^{m} f\right\|_{L_{p}\left(\Omega_{m, h}\right)} & \leq\left\|\Delta_{h}^{m} u\right\|_{L_{p}\left(\Omega_{m, h}\right)}+\left\|\Delta_{h}^{m} v\right\|_{L_{p}\left(\Omega_{m, h}\right)} \\
& \leq 2^{m}\|u\|_{L_{p}(\Omega)}+C|h|^{m}\|v\|_{W_{p}^{m}(\Omega)} \\
& \leq C \mathbb{K}\left(|h|^{m}, f\right) .
\end{aligned}
$$

In view of $\sigma=m \theta$ we get $|h|^{-\sigma}\left\|\Delta_{h}^{m} f\right\|_{L_{p}\left(\Omega_{m, h}\right)} \in L_{q}^{*}\left(\mathbb{R}^{d}\right)$ from $t^{-\theta} \mathbb{K}(t, f) \in L_{q}^{*}\left(\mathbb{R}_{+}\right)$ by the change of variables $h=t^{1 / m} \omega$ with $t>0$ and $|\omega|=1$. This combined with (9.1) yields (9.8) with

$$
\|f\|_{L_{p}(\Omega)}+|f|_{B_{p q}^{m, \sigma}(\Omega)} \leq C\|f\|_{\left(L_{p}(\Omega), W_{p}^{m}(\Omega)\right)_{\theta, q}} .
$$

Step 2. We shall show that

$$
B_{p q}^{m, \sigma}(\Omega) \subset B_{p q}^{\sigma}(\Omega)
$$

Let $f \in B_{p q}^{m, \sigma}(\Omega)$. We write Muramatu's formula (2.11) with $\lambda=1$ as $f=u+v$ with

$$
u=\int_{0}^{1} K_{t} * f \frac{d t}{t}, \quad v=\varphi * f
$$

where $K \in \mathcal{K}_{\Gamma}^{m}$. We have $v^{(\alpha)}=\varphi^{(\alpha)} * f \in L_{p}(\Omega)$ for all $\alpha \in \mathbb{N}_{0}^{d}$. This implies $v^{(\alpha)} \in W_{p}^{2}(\Omega)$ for $|\alpha|=[\sigma]_{-}$. Since $W_{p}^{2}(\Omega) \subset B_{p q}^{\{\sigma\}_{+}}(\Omega)$ by Lemma 9.7 , we see that $v \in B_{p q}^{\sigma}(\Omega)$ with $\|v\|_{B_{p q}^{\sigma}(\Omega)} \leq C\|v\|_{W_{p}^{[\sigma]_{-}+2}(\Omega)} \leq C\|f\|_{L_{p}(\Omega)}$.

As for $u$, we write $\partial^{\alpha}\left(K_{t} * f\right)=t^{-|\alpha|}\left(K^{(\alpha)}\right)_{t} * f$ and apply Lemma 9.8 to get

$$
\left\|\partial^{\alpha}\left(K_{t} * f\right)\right\|_{L_{p(\Omega)}} \leq t^{-|\alpha|+\sigma} G_{\alpha}(t)
$$

with some $G_{\alpha} \in L_{q}^{*}\left(\mathbb{R}_{+}\right)$satisfying $\left\|G_{\alpha}\right\|_{L_{q}^{*}\left(\mathbb{R}_{+}\right)} \leq C|f|_{B_{p q}^{m, \sigma}(\Omega)}$. If $|\alpha| \leq[\sigma]_{-}$, then Hölder's inequality gives

$$
\int_{0}^{1}\left\|\partial^{\alpha}\left(K_{t} * f\right)\right\|_{L_{p}(\Omega)} \frac{d t}{t} \leq\left\|t^{\sigma-[\sigma]_{-}}\right\|_{L_{q^{\prime}}^{*}(I)}\left\|G_{\alpha}\right\|_{L_{q}^{*}\left(\mathbb{R}_{+}\right)}<\infty
$$

with $1 / q+1 / q^{\prime}=1$ and $I=(0,1)$. By Proposition 2.9 we know that $\left.u \in W_{p}^{[\sigma]}\right]_{-}(\Omega)$ with

$$
u^{(\alpha)}=\int_{0}^{1} t^{-|\alpha|}\left(K^{(\alpha)}\right)_{t} * f \frac{d t}{t}, \quad \text { for }|\alpha| \leq[\sigma]_{-} .
$$

Next we want to evaluate $\Delta_{h}^{i} u^{(\alpha)}$ for $|\alpha|=[\sigma]_{-}$, where $i=1$ if $0<\{\sigma\}_{+}<1$, and $i=2$ if $\{\sigma\}_{+}=1$. Applying $\Delta_{h}^{i}$ to $(9.10)$ and using Lemma 9.8 for $\Delta_{h}^{i}\left(\left(K^{(\alpha)}\right)_{t} * f\right)$, we get

$$
\frac{\left\|\Delta_{h}^{i} u^{(\alpha)}\right\|_{L_{p}\left(\Omega_{i, h}\right)}}{|h|^{\{\sigma\}_{+}}} \leq \int_{0}^{1}\left(\frac{t}{|h|}\right)^{\{\sigma\}_{+}} \min \left\{1,\left(\frac{|h|}{t}\right)^{i}\right\} G(t) \frac{d t}{t}=: I(h)
$$

with some $G \in L_{q}^{*}\left(\mathbb{R}_{+}\right)$satisfying $\|G\|_{L_{q}^{*}\left(\mathbb{R}_{+}\right)} \leq C|f|_{B_{p q}^{m, \sigma}(\Omega)}$. Since it follows from $\{\sigma\}_{+}<i$ that $\int_{0}^{\infty} t^{\{\sigma\}_{+}} \min \left\{1, t^{-i}\right\} t^{-1} d t<\infty$, the map $G(t) \mapsto I(h)$ is a bounded 
operator from $L_{q}^{*}\left(\mathbb{R}_{+}\right)$to $L_{q}^{*}\left(\mathbb{R}^{d}\right)$ by Lemma 5.3. Therefore $u^{(\alpha)} \in B_{p q}^{\{\sigma\}_{+}}(\Omega)$ for $|\alpha|=[\sigma]_{-}$.

Combining the above estimates for $u$ and $v$, we obtain (9.9) with

$$
\|f\|_{B_{p q}^{\sigma}(\Omega)} \leq C\|f\|_{L_{p}(\Omega)}+|f|_{B_{p q}^{m, \sigma}(\Omega)} .
$$

Step 3. We shall show that

$$
B_{p q}^{\sigma}(\Omega) \subset\left(L_{p}(\Omega), W_{p}^{m}(\Omega)\right)_{\theta, q} .
$$

Let $f \in B_{p q}^{\sigma}(\Omega)$. We write Muramatu's formula (2.14) as $f=u+v$ with

$$
u=\sum_{|\beta|=[\sigma]_{-}} \int_{0}^{\lambda} t^{[\sigma]_{-}}\left(K_{\beta}\right)_{t} * f^{(\beta)} \frac{d t}{t}, \quad v=\varphi_{\lambda} * f,
$$

where $K_{\beta} \in \mathcal{K}_{\Gamma}^{2}$. Let $i=1$ if $0<\{\sigma\}_{+}<1$, and $i=2$ if $\{\sigma\}_{+}=1$. Since $f^{(\beta)} \in B_{p q}^{\{\sigma\}_{+}}(\Omega)=B_{p q}^{i,\{\sigma\}_{+}}(\Omega)$ for $|\beta|=[\sigma]_{-}$by Lemma 9.8, there exists $G \in$ $L_{q}^{*}\left(\mathbb{R}_{+}\right)$such that $\left\|\left(K_{\beta}\right)_{t} * f^{(\beta)}\right\|_{L_{p}(\Omega)} \leq t^{\{\sigma\}_{+}} G(t)$ for $|\beta|=[\sigma]_{-}$, and $\|G\|_{L_{q}^{*}\left(\mathbb{R}_{+}\right)} \leq$ $C \sum_{|\beta|=[\sigma]_{-}}\left|f^{(\beta)}\right|_{B_{p q}^{i,\{\sigma\}_{+}(\Omega)}}=C|f|_{B_{p q}^{\sigma}(\Omega)}$. Setting $G_{1}(\lambda)=\int_{0}^{\lambda}(t / \lambda)^{\sigma} G(t) t^{-1} d t$, we have

$$
\|u\|_{L_{p}(\Omega)} \leq C \int_{0}^{\lambda} t^{\sigma} G(t) \frac{d t}{t}=C \lambda^{\sigma} G_{1}(\lambda) .
$$

Also, $\left\|G_{1}\right\|_{L_{q}^{*}\left(\mathbb{R}_{+}\right)} \leq C\|G\|_{L_{q}^{*}\left(\mathbb{R}_{+}\right)}$by Lemma 5.3, and thereby $\left\|G_{1}\right\|_{L_{q}^{*}\left(\mathbb{R}_{+}\right)} \leq C|f|_{B_{p q}^{\sigma}(\Omega)}$.

We turn to estimates of $v$. By Young's inequality we have $\|v\|_{L_{p}(\Omega)} \leq C\|f\|_{L_{p}(\Omega)}$. For $|\alpha|=m$ we take $\beta$ so that $\beta \leq \alpha$ and $|\beta|=[\sigma]_{-}$, and write

$$
v^{(\alpha)}=\lambda^{[\sigma]_{-}-m}\left(\varphi^{(\alpha-\beta)}\right)_{\lambda} * f^{(\beta)} .
$$

By Lemma 9.8 we have $\left\|v^{(\alpha)}\right\|_{L_{p}(\Omega)} \leq \lambda^{\sigma-m} G_{2}(\lambda)$ with some $G_{2} \in L_{q}^{*}\left(\mathbb{R}_{+}\right)$satisfying $\left\|G_{2}\right\|_{L_{q}^{*}\left(\mathbb{R}_{+}\right)} \leq C|f|_{B_{p q}^{\sigma}(\Omega)}$.

The interpolation inequality and the estimates for $u$ and $v$ give

$$
\begin{aligned}
\mathbb{K}\left(\lambda^{m}, f\right) & \leq\|u\|_{L_{p}(\Omega)}+\lambda^{m}\|v\|_{W_{p}^{m}(\Omega)} \\
& \leq C\left\{\lambda^{\sigma} G_{1}(\lambda)+\lambda^{m}\|f\|_{L_{p}(\Omega)}+\lambda^{\sigma} G_{2}(\lambda)\right\} .
\end{aligned}
$$

We use this inequality for $0<\lambda \leq 1$, and the inequality $\mathbb{K}\left(\lambda^{m}, f\right) \leq\|f\|_{L_{p}}$, which follows from $f=f+0 \in L_{p}(\Omega)+W_{p}^{m}(\Omega)$, for $\lambda>1$. Then we know $\lambda^{-\sigma} \mathbb{K}\left(\lambda^{m}, f\right) \in L_{q}^{*}\left(\mathbb{R}_{+}\right)$and thereby $t^{-\theta} \mathbb{K}(t, f) \in L_{q}^{*}\left(\mathbb{R}_{+}\right)$. We obtain (9.11) with $\|f\|_{\left(L_{p}(\Omega), W_{p}^{m}(\Omega)\right)_{\theta, q}} \leq C\|f\|_{B_{p q}^{\sigma}(\Omega)}$.

\section{Sobolev spaces of negative order}

Muramatu's integral formula also works for Sobolev spaces of negative order. Before giving its definition we collect here the basic facts on distribution theory. A distribution $T$ on $\Omega$ is a continuous linear functional on $C_{0}^{\infty}(\Omega)$ in the sense that for every 
compact subset $K$ of $\Omega$ there exist $k \in \mathbb{N}_{0}$ and a constant $C>0$ such that

$$
\left|\langle\phi, T\rangle_{\Omega}\right| \leq C \sum_{|\alpha| \leq k}\left\|\partial^{\alpha} \phi\right\|_{L_{\infty}(\Omega)}, \quad \text { for all } \phi \in C_{0}^{\infty}(\Omega) \text { with } \operatorname{supp} \phi \subset K .
$$

Here we denote the value of $T$ at $\phi \in C_{0}^{\infty}(\Omega)$ by $\langle\phi, T\rangle_{\Omega}$. A locally integrable function $f(x)$ is considered as a distribution by $\langle\phi, f\rangle_{\Omega}=\int_{\Omega} \phi(x) f(x) d x$. The derivative $\partial^{\alpha} T$ is defined by

$$
\left\langle\phi, \partial^{\alpha} T\right\rangle_{\Omega}=(-1)^{|\alpha|}\left\langle\partial^{\alpha} \phi, T\right\rangle_{\Omega},
$$

and the product $\zeta T$ with $\zeta \in C^{\infty}(\Omega)$ is defined by

$$
\langle\phi, \zeta T\rangle_{\Omega}=\langle\zeta \phi, T\rangle_{\Omega}
$$

It is sometimes helpful to write a distribution $T$ as $T(x)$ with variable $x$ attached, even when $T$ is not actually a function. Recall that $\Gamma$ is the cone satisfying (2.2) for $\Omega$. If $\phi \in C_{0}^{\infty}\left(\mathbb{R}^{d}\right)$ satisfies $\operatorname{supp} \phi \subset-\Gamma$, then we can define the convolution $\phi * T$ by

$$
\phi * T(x)=\langle\phi(x-\cdot), T\rangle_{\Omega}=\langle\phi(x-y), T(y)\rangle_{\Omega}
$$

for $x \in \Omega$, since $\operatorname{supp} \phi(x-\cdot) \subset x+\Gamma \subset \Omega$.

The following lemma tells us how to define Sobolev spaces of negative order.

Lemma 10.1. Let $1<p<\infty, m \in \mathbb{N}_{0}$ and $k \in \mathbb{N}$. Then the following conditions are equivalent:

(a) $f \in W_{p}^{m}(\Omega)$.

(b) There exist $f_{\alpha} \in W_{p}^{m+k}(\Omega)$ with $|\alpha| \leq k$ such that $f$ is written as $f=$ $\sum_{|\alpha| \leq k} \partial^{\alpha} f_{\alpha}$

Proof. By definition of Sobolev spaces (b) $\Rightarrow(\mathrm{a})$ is obvious. To show (a) $\Rightarrow(\mathrm{b})$ we use Muramatu's formula (2.14) with $\lambda=1, K_{\beta}=\sum_{|\alpha|=k} H_{\alpha \beta}^{(\alpha)}$ and $H_{\alpha \beta} \in \mathcal{K}_{\Gamma}^{1}$. Since $\left(H_{\alpha \beta}^{(\alpha)}\right)_{t}=t^{|\alpha|} \partial^{\alpha}\left(H_{\alpha \beta}\right)_{t}$, Corollary 6.2 gives

$$
f=\sum_{|\alpha|=k} \sum_{|\beta|=m} \partial^{\alpha} \int_{0}^{1} t^{m+k}\left(H_{\alpha \beta}\right)_{t} * f^{(\beta)} \frac{d t}{t}+\varphi * f .
$$

Define $f_{\alpha}$ by $f_{\alpha}=\varphi * f$ for $\alpha=0, f_{\alpha}=0$ for $0<|\alpha|<k$, and

$$
f_{\alpha}=\sum_{|\beta|=m} \int_{0}^{1} t^{m+k}\left(H_{\alpha \beta}\right)_{t} * f^{(\beta)} \frac{d t}{t}, \quad \text { for }|\alpha|=k .
$$

Then these $f_{\alpha}$ satisfy the desired conditions in (b).

Definition 10.2. Let $1 \leq p \leq \infty, m \in \mathbb{Z}$ and $m<0$. The Sobolev space $W_{p}^{m}(\Omega)$ is the collection of all distributions $f$ which are written as

$$
f=\sum_{|\alpha| \leq-m} \partial^{\alpha} f_{\alpha}, \quad f_{\alpha} \in L_{p}(\Omega)
$$


in the distributional sense. We equip $W_{p}^{m}(\Omega)$ with the norm

$$
\|f\|_{W_{p}^{m}(\Omega)}=\inf \sum_{|\alpha| \leq-m}\left\|f_{\alpha}\right\|_{L_{p}(\Omega)},
$$

where the infimum is taken over all the expressions of $f$ in (10.1).

It is not difficult to show that the Sobolev space $W_{p}^{m}(\Omega)$ of negative order is also a Banach space, using the completeness of $L_{p}(\Omega)$ and the inequalities

$$
\left\|\partial^{\alpha} f\right\|_{W_{p}^{m}(\Omega)} \leq\|f\|_{L_{p}(\Omega)}, \quad \text { for } f \in L_{p}(\Omega),|\alpha| \leq-m .
$$

By definition we see that the following embedding is continuous:

$$
W_{p}^{m}(\Omega) \subset W_{p}^{n}(\Omega), \quad \text { for } m>n \text { with } m, n \in \mathbb{Z},
$$

where $\mathbb{Z}$ is the set of integers.

Let $m$ be a negative integer and let $f \in W_{p}^{m}(\Omega)$ be written as (10.1). We take a function $\varphi \in \mathcal{K}_{\Gamma}^{0}$, and consider the convolution $\varphi_{t} * f(x)$ for $t>0$. Since

$$
\begin{aligned}
\left\langle\varphi_{t}(x-y), \partial^{\alpha} f_{\alpha}(y)\right\rangle_{\Omega} & =(-1)^{|\alpha|}\left\langle\partial_{y}^{\alpha}\left\{\varphi_{t}(x-y)\right\}, f_{\alpha}(y)\right\rangle_{\Omega} \\
& =\int_{\Omega} \partial_{x}^{\alpha}\left\{\varphi_{t}(x-y)\right\} f_{\alpha}(y) d y
\end{aligned}
$$

for $x \in \Omega$, we see that $\varphi_{t} * f(x) \in C^{\infty}\left(\mathbb{R}_{+} \times \Omega\right)$ and that

$$
\begin{aligned}
\partial_{t}^{k} \partial_{x}^{\beta}\left(\varphi_{t} * f(x)\right) & =\sum_{|\alpha| \leq-m} \int_{\Omega} \partial_{t}^{k} \partial_{x}^{\alpha+\beta}\left\{\varphi_{t}(x-y)\right\} f_{\alpha}(y) d y \\
& =\sum_{|\alpha| \leq-m} \partial_{t}^{k} \partial_{x}^{\alpha+\beta}\left(\varphi_{t} * f_{\alpha}(x)\right)
\end{aligned}
$$

for $k \in \mathbb{N}_{0}, \beta \in \mathbb{N}_{0}^{d}$. As counterparts of Theorems 2.11 and 2.5 for non-negative orders, we formulate the two theorems for negative orders.

Theorem 10.3. Let $1 \leq p<\infty, m \in \mathbb{Z}$ and $m<0$. Then $W_{p}^{m}(\Omega) \cap C^{\infty}(\bar{\Omega})$ is dense in $W_{p}^{m}(\Omega)$.

Proof. First we assume $f \in L_{p}(\Omega)$ and consider $u(t, x)=\left\langle\varphi_{t}(x-y), \partial^{\alpha} f(y)\right\rangle_{\Omega}$ for $x \in \Omega, t>0$ and $\varphi \in \mathcal{K}_{\Gamma}^{0}$ satisfying $\int_{\mathbb{R}^{d}} \varphi(x) d x=1$. Let $\epsilon>0$ and set

$$
\delta=\operatorname{dist}\left(-\operatorname{supp} \varphi, \Gamma^{c}\right)>0, \quad \Omega^{\epsilon}=\left\{x \in \mathbb{R}^{d}: \operatorname{dist}(x, \Omega)<\epsilon\right\} .
$$

We shall show that $u(t, x)$ is also defined for $x \in \Omega^{t \delta}$, that $u(t, \cdot) \in C^{\infty}\left(\Omega^{t \delta}\right)$ and that $u(t, \cdot) \rightarrow \partial^{\alpha} f$ in $W_{p}^{-|\alpha|}(\Omega)$ as $t \rightarrow 0$.

Let $x \in \Omega^{t \delta}$. Then there is $x_{0} \in \Omega$ such that $\left|x-x_{0}\right|<t \delta$, and $\varphi_{t}(x-y) \neq 0$ implies

$$
y \in x+t(-\operatorname{supp} \varphi)=x_{0}+t\left(\left(x-x_{0}\right) / t-\operatorname{supp} \varphi\right) \subset x_{0}+t \Gamma \subset \Omega .
$$


Therefore $\varphi_{t}(x-\cdot) \in C_{0}^{\infty}(\Omega)$, and

$$
\begin{aligned}
u(t, x) & =(-1)^{-|\alpha|}\left\langle\partial_{y}^{\alpha} \varphi_{t}(x-y), f(y)\right\rangle_{\Omega} \\
& =\left\langle\partial_{x}^{\alpha} \varphi_{t}(x-y), f(y)\right\rangle_{\Omega} \\
& =\partial^{\alpha}\left\{\varphi_{t} * E_{0} f(x)\right\}
\end{aligned}
$$

for $x \in \Omega^{t \delta}$, where $E_{0} f$ is the zero-extension to $\mathbb{R}^{d}$ defined by (2.9). It follows from $\varphi_{t} * E_{0} f \in C^{\infty}\left(\mathbb{R}^{d}\right)$ that $u(t, x) \in C^{\infty}\left(\Omega^{t \delta}\right)$. Since $\varphi_{t} * f \rightarrow f$ in $L_{p}(\Omega)$, we have

$$
\begin{aligned}
\left\|u(t, \cdot)-\partial^{\alpha} f\right\|_{W_{p}^{-|\alpha|}(\Omega)} & =\left\|\partial^{\alpha}\left\{\varphi_{t} * f-f\right\}\right\|_{W_{p}^{-|\alpha|}(\Omega)} \\
& \leq\left\|\varphi_{t} * f-f\right\|_{L_{p}(\Omega)} \rightarrow 0
\end{aligned}
$$

as $t \rightarrow 0$. Thus we have shown the assertion for $u(t, x)$.

The theorem follows by writing $f \in W_{p}^{m}(\Omega)$ in the form of (10.1), and applying the above assertion to $f_{\alpha} \in L_{p}(\Omega)$ in $(10.1)$.

Theorem 10.4. Let $1 \leq p<\infty, m \in \mathbb{Z}, m<0, N \in \mathbb{N}$ and $\lambda>0$. Then there exist $K \in \mathcal{K}_{\Gamma}^{N}$ and $\varphi \in \mathcal{K}_{\Gamma}^{0}$ satisfying $\int_{\Omega} \varphi(x) d x=1$ such that

$$
f=\sum_{|\alpha| \leq-m} \partial^{\alpha} \int_{0}^{\lambda} K_{t} * f_{\alpha} \frac{d t}{t}+\sum_{|\alpha| \leq-m} \lambda^{-|\alpha|}\left(\varphi^{(\alpha)}\right)_{\lambda} * f_{\alpha}
$$

holds for $f \in W_{p}^{m}(\Omega)$ written as (10.1), where the integral $\int_{0}^{\lambda}$ should be interpreted as the limit of $\int_{\epsilon}^{\lambda}$ as $\epsilon \rightarrow 0$ in the norm of $L_{p}(\Omega)$.

Proof. We take $\varphi$ and $K$ as in Lemma 2.3. By (2.8)

$$
\varphi_{\epsilon} * f_{\alpha}=\int_{\epsilon}^{\lambda} K_{t} * f_{\alpha} \frac{d t}{t}+\varphi_{\lambda} * f_{\alpha}
$$

for $0<\epsilon<\lambda$. Applying $\partial^{\alpha}$ to (10.4), and summing over $|\alpha| \leq-m$, we get, by $(10.2)$

$$
\varphi_{\epsilon} * f=\sum_{|\alpha| \leq-m} \partial^{\alpha} \int_{\epsilon}^{\lambda} K_{t} * f_{\alpha} \frac{d t}{t}+\sum_{|\alpha| \leq-m} \lambda^{-|\alpha|}\left(\varphi^{(\alpha)}\right)_{\lambda} * f_{\alpha} .
$$

Since $\varphi_{\epsilon} * f \rightarrow f$ in $W_{p}^{m}(\Omega)$ as $\epsilon \rightarrow 0$, as shown in the proof of Theorem 10.3, and since (10.4) gives $\int_{\epsilon}^{\lambda} K_{t} * f_{\alpha} t^{-1} d t \rightarrow f_{\alpha}-\varphi_{\lambda} * f_{\alpha}$ in $L_{p}(\Omega)$, we obtain the theorem.

Remark 10.5. Let $f \in L_{p}(\Omega), K \in \mathcal{K}_{\Gamma}^{1}$ and $\lambda>0$. We have already known that the integral $\int_{0}^{\lambda} K_{t} * f t^{-1} d t$ converges in $L_{p}(\Omega)$ if $p>1$ by Theorem 6.1. As for the case $p=1$, the integral converges in the norm of $W_{p}^{-1}(\Omega)$. Indeed, writing $K=\sum_{|\beta|=1} \partial^{\beta} K_{\beta}$ with $K_{\beta} \in \mathcal{K}_{\Gamma}^{0}$, we have $\int_{\epsilon}^{\lambda} K_{t} * f t^{-1} d t=\sum_{|\beta|=1} \partial^{\beta} \int_{\epsilon}^{\lambda}\left(K_{\beta}\right)_{t} * f d t$ for $\epsilon>0$, and know that $\int_{\epsilon}^{\lambda}\left(K_{\beta}\right)_{t} * f d t$ converges in $L_{1}(\Omega)$ as $\epsilon \rightarrow 0$ by Proposition 2.9 ; hence $\partial^{\beta} \int_{\epsilon}^{\lambda}\left(K_{\beta}\right)_{t} * f d t$ converges in $W_{1}^{-1}(\Omega)$. Also, we have $\int_{0}^{\lambda} K_{t} * f t^{-1} d t=$ $\sum_{|\beta|=1} \partial^{\beta} \int_{0}^{\lambda}\left(K_{\beta}\right)_{t} * f d t$ 
As a consequence, if $K \in \mathcal{K}_{\Gamma}^{0}$ then

$$
\partial_{j} \int_{0}^{\lambda} K_{t} * f d t=\int_{0}^{\lambda} \partial_{j}\left(K_{t} * f\right) d t=\int_{0}^{\lambda}\left(\partial_{j} K\right)_{t} * f \frac{d t}{t},
$$

where the integral on the right-hand side should be regarded as the limit of $\int_{\epsilon}^{\lambda}$ in $W_{1}^{-1}(\Omega)$ as $\epsilon \rightarrow 0$. This fact will be used in the proof of Theorem 10.6 below.

Theorem 10.6. Let $1 \leq p<q<\infty, m, n \in \mathbb{Z}$ and $m-d / p \geq n-d / q$. In addition, we assume that $m-d / p>n-d / q$ if $p=1$. Then $W_{p}^{m}(\Omega) \subset W_{q}^{n}(\Omega)$, and the inequality

$$
\|f\|_{W_{q}^{n}(\Omega)} \leq C\|f\|_{W_{p}^{m}(\Omega)}, \quad \text { for } f \in W_{p}^{m}(\Omega)
$$

holds with $C=C(m, n, p, q, d, \Omega)$.

Proof. We note that $m>n$ since $m-n \geq d(1 / p-1 / q)>0$. Since we have already treated the case $m \in \mathbb{N}$ and $n \in \mathbb{N}_{0}$ in Theorem 3.1, it remains to consider the other cases.

Case $m \geq 0$ and $n<0$. Let $f \in W_{p}^{m}(\Omega)$. We use Muramatu's formula (2.14) with $\lambda=1, K_{\beta}=\sum_{|\alpha|=-n} \partial^{\alpha} K_{\alpha \beta}$ and $K_{\alpha \beta} \in \mathcal{K}_{\Gamma}^{1}$. By Corollary 6.2 for $p>1$, and by Proposition 2.9 and Remark 10.5 for $p=1$, we have

$$
f=\sum_{|\beta|=m} \sum_{|\alpha|=-n} \partial^{\alpha} \int_{0}^{1} t^{m-n}\left(K_{\alpha \beta}\right)_{t} * f^{(\beta)} \frac{d t}{t}+\varphi * f .
$$

We can evaluate the integral $F_{\alpha \beta}=\int_{0}^{1} t^{m-n}\left(K_{\alpha \beta}\right)_{t} * f^{(\beta)} t^{-1} d t$ as in the proof of Theorem 3.1 for Cases 1 and 2 to get

$$
\|f\|_{W_{q}^{n}} \leq \sum_{|\beta|=m} \sum_{|\alpha|=-n}\left\|F_{\alpha \beta}\right\|_{L_{q}}+\|\varphi * f\|_{L_{q}} \leq C \sum_{|\beta|=m}\left\|f^{(\beta)}\right\|_{L_{p}}+\|\varphi\|_{L_{r}}\|f\|_{L_{p}}
$$

with $p^{-1}+r^{-1}=1+q^{-1}$. This implies $f \in W_{q}^{n}(\Omega)$ and $\|f\|_{W_{q}^{n}} \leq C\|f\|_{W_{p}^{m}}$.

Case $m<0$ and $n<0$. Let $f \in W_{p}^{m}(\Omega)$. We use (10.3) with $\lambda=1, K=$ $\sum_{|\beta|=m-n} \partial^{\beta} K_{\beta}$ and $K_{\beta} \in \mathcal{K}_{\Gamma}^{1}$. Then

$$
f=\sum_{|\alpha| \leq-m} \sum_{|\beta|=m-n} \partial^{\alpha+\beta} \int_{0}^{1} t^{m-n}\left(K_{\beta}\right)_{t} * f_{\alpha} \frac{d t}{t}+\sum_{|\alpha| \leq-m} \varphi^{(\alpha)} * f_{\alpha} .
$$

Noting that $0 \leq|\alpha+\beta|=|\alpha|+m-n \leq-n$, we can evaluate $\|f\|_{W_{q}^{n}}$ similarly.

As an application of negative-order Sobolev spaces we show a lemma which is required for the regularity theorem for elliptic equations. We can see that Muramatu's formula works well in its proof.

Lemma 10.7. Let $1<p<\infty, m, k \in \mathbb{N}, m>k$ and $d \geq 2$. Suppose that $f \in L_{p}(\Omega)$ satisfies $\partial_{j}^{k} f \in L_{p}(\Omega)$ for $j=1, \ldots, d-1$ and $\partial_{d}^{m} f \in W_{p}^{k-m}(\Omega)$. Then we have $f \in W_{p}^{k}(\Omega)$ and

$$
\|f\|_{W_{p}^{k}(\Omega)} \leq C\left(\|f\|_{L_{p}(\Omega)}+\sum_{j=1}^{d-1}\left\|\partial_{j}^{k} f\right\|_{L_{p}(\Omega)}+\left\|\partial_{d}^{m} f\right\|_{W_{p}^{k-m}(\Omega)}\right)
$$


with $C=C(m, k, p, d, \Omega)$.

Proof. We write Muramatu's formula (2.11) with $\lambda=1$ as

$$
f=\int_{0}^{1} \sum_{|\alpha|=m d}\left(K_{\alpha}^{(\alpha)}\right)_{t} * f \frac{d t}{t}+\varphi * f
$$

with $K_{\alpha} \in \mathcal{K}_{\Gamma}^{1}$. Since $|\alpha|=m d$ implies $\alpha_{j} \geq m(>k)$ for some $j=1, \ldots, d$, we have

$$
f=\int_{0}^{1} \sum_{j=1}^{d-1} t^{k}\left(K_{j}\right)_{t} * \partial_{j}^{k} f \frac{d t}{t}+\int_{0}^{1} t^{m}\left(K_{d}\right)_{t} * \partial_{d}^{m} f \frac{d t}{t}+\varphi * f
$$

with some $K_{j} \in \mathcal{K}_{\Gamma}^{1}$ for $j=1, \ldots, d$. By assumption, $\partial_{d}^{m} f$ is written as $\partial_{d}^{m} f=$ $\sum_{|\beta| \leq m-k} \partial^{\beta} f_{\beta}$ with $f_{\beta} \in L_{p}(\Omega)$. Substituting this expression in the above equation, and using $\left(K_{d}\right)_{t} * \partial^{\beta} f_{\beta}=t^{-|\beta|}\left(K_{d}^{(\beta)}\right)_{t} * f_{\beta}$, we have

$$
f=\sum_{j=1}^{d-1} \int_{0}^{1} t^{k}\left(K_{j}\right)_{t} * \partial_{j}^{k} f \frac{d t}{t}+\sum_{|\beta| \leq m-k} \int_{0}^{1} t^{m-|\beta|}\left(K_{d}^{(\beta)}\right)_{t} * f_{\beta} \frac{d t}{t}+\varphi * f .
$$

By Theorem 6.1 and Corollary 6.2 we get $f \in W_{p}^{k}(\Omega)$ with

$$
\|f\|_{W_{p}^{k}(\Omega)} \leq C\left(\sum_{j=1}^{d-1}\left\|\partial_{j}^{k} f\right\|_{L_{p}(\Omega)}+\sum_{|\beta| \leq m-k}\left\|f_{\beta}\right\|_{L_{p}(\Omega)}+\|f\|_{L_{p}(\Omega)}\right) .
$$

The desired inequality follows by taking the infimum.

\section{Concluding remarks}

\subsection{Muramatu's work}

It is known that most function spaces are covered by two scales of function spaces, the Triebel-Lizorkin space $F_{p q}^{\sigma}(\Omega)$ and the Besov space $B_{p q}^{\sigma}(\Omega)$. For example, $W_{p}^{\sigma}(\Omega)=$ $F_{p 2}^{\sigma}(\Omega)$ and $\mathcal{C}^{\sigma}(\Omega)=B_{\infty \infty}^{\sigma}(\Omega)$. For these generalized spaces we refer to $[26,36,37$, $38,39]$.

In addition to [22], Muramatu's work concerning integral formulas is found in $[23,24,25]$. The paper [23] is an expository article for [22], and exemplifies the usefulness of Muramatu's formula by applying it to the proof of the embedding theorem $W_{p}^{m}\left(\mathbb{R}_{+}^{d}\right) \subset C^{k}\left(\mathbb{R}_{+}^{d}\right)$ for $m-d / p>k$ and the interpolation inequality (Corollary 2.10). In a chapter of the textbook [24] he developed the theory of $L_{2^{-}}$ based Sobolev spaces $W_{2}^{\sigma}(\Omega)$ of fractional order by the second integral formula, and applied it to elliptic boundary value problems and hyperbolic equations. In the book [25] he investigated interpolation spaces with function parameter and applied it to the Besov space $B_{p q}^{\psi}\left(\mathbb{R}^{d}\right)$ with function parameter by the second integral formula. Here $\psi$ is a function on $\mathbb{R}_{+}$which generalizes the function $t \mapsto t^{\sigma}$ with a fixed $\sigma \in \mathbb{R}$. When $\psi(t)=t^{\sigma}$, we have the relation $B_{p q}^{\psi}\left(\mathbb{R}^{d}\right)=B_{p q}^{\sigma}\left(\mathbb{R}^{d}\right)$.

In this paper as well as in Muramatu's work, the condition on $p$ varies from theorem to theorem within the range $1 \leq p \leq \infty$. Roughly speaking, we must assume 
$1 \leq p<\infty$ if the limit $\varphi_{\epsilon} * f \rightarrow f$ in $L_{p}(\Omega)$ as $\epsilon \rightarrow 0$ is concerned, and $1<p<\infty$ if Theorem 6.1, which is based on the Calderón-Zygmund theory, is required. In the trace theorem we assumed $1<p<\infty$ since the limit $\varphi_{\epsilon} * f$ and the integral $\int_{0}^{1} t^{-1 / p} d t$ are concerned.

\subsection{Approximation by $C^{\infty}$ functionss}

It is known that $W_{p}^{m}(\Omega) \cap C^{\infty}(\Omega)$ is dense in $W_{p}^{m}(\Omega)$ for an arbitrary domain $\Omega$ by Meyers-Serrin's theorem (see [1, Theorem 3.17]). Theorem 2.11 and Theorem 10.3 make a stronger assumption that $\Omega$ satisfies the cone condition $(2,2)$, so these theorems conclude more strongly that $W_{p}^{m}(\Omega) \cap C^{\infty}(\bar{\Omega})$ is dense in $W_{p}^{m}(\Omega)$; note that a function $f \in C^{\infty}(\Omega)$ does not necessarily extend to a function belonging to $C^{\infty}(\bar{\Omega})$.

\subsection{Integral representations}

There are several integral representation formulas for functions in Sobolev spaces. Compared with Sobolev's representation formula (see [2, Theorem 11.5] and [7, Chapter 3]), Muramatu's formula has the advantage that it works not only for functions but also for distributions, as Muramatu states in [23]. The integral representation formula which Besov-Il'in-Nikol'skii [4,5] devised for anisotropic Sobolev spaces is similar to Muramatu's formula, since both formulas are based on the integration of $\partial_{t}\left(\varphi_{t} * f(x)\right)$ over the interval $[\epsilon, \lambda]$. But the integral kernels are constructed in different ways.

Rychkov [27] used a reproducing formula

$$
f=\sum_{j=0}^{\infty} \psi_{j} * \kappa_{j} * f
$$

to construct an extension operator for the Triebel-Lizorkin and Besov spaces. Formula (11.1) is derived from $f=\sum_{j=0}^{\infty} \kappa_{j} * f$, which is considered as a discrete version of Muramatu's formula. In our terminology it is written as

$$
f=\varphi * f+\sum_{j=1}^{\infty}\left(\varphi_{2^{-j}}-\varphi_{2^{1-j}}\right) * f
$$

where $\varphi$ is the function in Theorem 2.5 and $\varphi_{2^{-j}}(x)=2^{j d} \varphi\left(2^{j} x\right)$.

When $\Omega=\mathbb{R}^{d}$, we can choose a function $\varphi$ in Muramatu's formula from the Schwartz space $\mathcal{S}\left(\mathbb{R}^{d}\right)$, since we do not have to pay attention to the support of $\varphi$. If we set $\varphi(x)=\mathcal{F}^{-1}\left[e^{-P(\xi)}\right](x)$ for a homogeneous polynomial $P(\xi)$ of even order $k$ satisfying $P(\xi)>0$ for $\xi \neq 0$, then $\varphi \in \mathcal{S}\left(\mathbb{R}^{d}\right)$, and $K$ defined by (2.6) or rather $(2.7)$ is written as

$$
K(x)=k \mathcal{F}^{-1}\left[P(\xi) e^{-P(\xi)}\right]=k(2 \pi i)^{-k} P(\partial / \partial x)\{\varphi(x)\}
$$




\subsection{Sobolev's embeddings and traces}

The proof of Theorem 3.1 for the case $p>1$ and $m-d / p=n-d / q$ goes back to the proof due to Sobolev. In the standard way Sobolev's embedding theorem $W_{p}^{m}\left(\mathbb{R}^{d}\right) \rightarrow W_{q}^{n}\left(\mathbb{R}^{d}\right)$ (Theorem 3.1) is proved in the reverse order; the case $m-d / p>$ $n-d / q$ reduces to the case $m-d / p=n-d / q$. Tartar's approach [32, Chapter 30] to Sobolev's embedding theorem is interesting. He derived the inequality for the critical case $p=1, q=d /(d-1)$ and $d \geq 2$ from that for the case $p=1,1<q<d /(d-1)$ and $d \geq 2$. At the same time he elaborated the inequality in terms of Lorentz spaces.

Sobolev's inequality (3.4) and Nash's inequality (Theorem 3.2) are special cases of the Gagliardo-Nirenberg inequality, whose formulation and proof are found in [31, Chapter 3], [13, Section 1.8]. Equivalence between Sobolev's inequality and Nash's inequality is discussed in [28].

The proof of the existence and boundedness of the trace operator (Theorem $5.2)$ for the half space is essentially the same as one of the three proofs given in [20], which deals with the whole space. If we use the integral representation by the Gauss kernel instead of Muramatu's formula, we can give a proof of trace theorem for Sobolev spaces of fractional order on $\mathbb{R}^{d}$ (see [20]). The idea to prove the surjectivity of the trace operator (Corollary 5.4) by regularization goes back to Stein [29].

\subsection{Extensions}

The extension operator constructed in the proof of Theorem 7.1 is independent of $p$, but it depends on $m$. If we use Muramatu's second integral formula, we can construct an extension operator

$$
E: \bigcup_{k=-m}^{m} W_{p}^{k}(\Omega) \rightarrow \bigcup_{k=-m}^{m} W_{p}^{k}\left(\mathbb{R}^{d}\right)
$$

for a given $m \in \mathbb{N}$ so that the restriction of $E$ to $W_{p}^{k}(\Omega)$ is an extension from $W_{p}^{k}(\Omega) \rightarrow W_{p}^{k}\left(\mathbb{R}^{d}\right)$ for each $k$ with $-m \leq k \leq m$. Stein [30, Chapter 6] constructed an extension independent of $p \in[1, \infty]$ and $m \in \mathbb{N}_{0}$. For an extension in the more general settings we refer to Rychkov [27].

The idea of using the zero-extension to construct our extention operator comes from the proof of Calderón's extension theorem (see [2, Theorem 11.12], [1, Theorem $5.28]$ ), which is based on Sobolev's representation formula.

\subsection{Interpolation of Sobolev spaces}

By Theorem 8.2 it is reasonable to define Sobolev spaces of fractional order by

$$
W_{p}^{\sigma}(\Omega)=\left[W_{p}^{n}(\Omega), W_{p}^{m}(\Omega)\right]_{\theta}
$$

for $\sigma \in \mathbb{R} \backslash \mathbb{Z}$, if we take $m, n \in \mathbb{Z}$ and $\theta \in(0,1)$ so that $\sigma=(1-\theta) n+\theta m$. In [22] Muramatu showed Theorem 8.2 for Sobolev spaces of fractional order by characterizing Sobolev spaces in terms of $L_{p}\left(\Omega, L_{2}^{*}(I)\right)$ with $I=(0,1)$ and using the complex interpolation of the spaces related to $L_{p}\left(\Omega, L_{2}^{*}(I)\right)$. 
In the theory of real interpolation spaces there are two major methods, the $J$-method and the $K$-method. They are equivalent. See $[1,3,35]$ for the details of these methods.

In $[22,25]$ Muramatu proved Theorem 9.3 by the $J$-method, characterizing $B_{p q}^{\sigma}(\Omega)$ in terms of $L_{q}^{*}\left(I, L_{p}(\Omega)\right)$ with $I=(0,1)$ with the help of the second integral formula, and using the real interpolation of the spaces related to $L_{q}^{*}\left(I, L_{p}(\Omega)\right)$. Note that $f \in B_{p q}^{\sigma}(\Omega)$ implies $t^{-\sigma} K_{t} * f(x) \in L_{q}^{*}\left(\mathbb{R}_{+}, L_{p}(\Omega)\right)$ for $K \in \mathcal{K}_{\Gamma}^{m}$ by Theorem 9.3 and Lemma 9.8. Our proof using the $K$-method is inspired by the proof of $[1$, Theorem 7.47], where it is assumed that $m$ is even.

Lemma 9.5 is a key tool, when dealing with Besov spaces by Muramatu's integral formula. It was obtained by Muramatu in [25] when the domain is $\mathbb{R}^{d}$. Although the integral kernel $W_{t}(x-y, x-y-z)$ is used in [25], we must use $W_{t}(x-y, x-y-m z)$ in order to extend Lemma 9.5 to the domains different from $\mathbb{R}^{d}$.

It is known that $F_{p q}^{\sigma}\left(\mathbb{R}^{d}\right)=B_{p_{1} q_{1}}^{\sigma_{1}}\left(\mathbb{R}^{d}\right)$ if and only if $\sigma=\sigma_{1}$ and $p=p_{1}=q=q_{1}$ (see [36, Theorem 2.3.9]). Since $W_{p}^{\sigma}\left(\mathbb{R}^{d}\right)=F_{p 2}^{\sigma}\left(\mathbb{R}^{d}\right)$ as stated above, we know that $W_{p}^{\sigma}\left(\mathbb{R}^{d}\right)=B_{p p}^{\sigma}\left(\mathbb{R}^{d}\right)$ if and only if $p=2$. In other words, $W_{p}^{\sigma}\left(\mathbb{R}^{d}\right) \neq B_{p p}^{\sigma}\left(\mathbb{R}^{d}\right)$ if $p \neq 2$. There are some references, including [19] and [8], which write $W_{p}^{\sigma}(\Omega)$ for the Besov space $B_{p p}^{\sigma}(\Omega)$ with non-integer $\sigma$. One should be careful about the definition of $W_{p}^{\sigma}(\Omega)$ when reading such references.

\subsection{Sobolev spaces of negative order}

For Lemma 10.7 related to the regularity theorem for elliptic equations we refer to [2, Lemma 9.3], [13, Lemma 17.2], where $p=2$ is assumed, the domain is a half ball, and the condition $\partial_{d}^{m} f \in W_{p}^{k-m}(\Omega)$ is formulated in the form

$$
\left|\int_{\Omega} f(x) \partial_{d}^{m} \phi(x) d x\right| \leq M\|\phi\|_{W_{p^{\prime}}^{m-k}(\Omega)}, \quad \text { for all } \phi \in C_{0}^{\infty}(\Omega)
$$

with some constant $M>0$ and $1 / p+1 / p^{\prime}=1$. Since $W_{p}^{k-m}(\Omega)$ is the dual of the completion of $C_{0}^{\infty}(\Omega)$ in $W_{p^{\prime}}^{m-k}(\Omega)$ by [1, Theorem 3.12], estimate (11.2) is equivalent to our formulation in terms of negative-order Sobolev spaces.

\subsection{Sobolev spaces on a bounded domain}

In this paper, we have dealt with Sobolev spaces only on $\mathbb{R}^{d}$ or a special Lipschitz domain. In order to treat a bounded Lipschitz domain by Muramatu's method, we start from a modified regularization of the form

$$
\int_{\Omega} t^{-d} \varphi\left(\frac{x-y}{t}+\psi(x)\right) f(y) d y
$$

in place of $\varphi_{t} * f(x)$, where $\varphi(x)$ is a $C^{\infty}$ function supported in the unit ball $B$, and $\psi(x)$ is an $\mathbb{R}^{d}$-valued $C^{\infty}$ function on $\bar{\Omega}$ which satisfies

$$
x+t(\psi(x)+B) \subset \Omega, \quad \text { for all } x \in \Omega \text { and all } t \in\left(0, t_{\Omega}\right]
$$

with a suitable constant $t_{\Omega}$. For a bounded Lipschitz domain we can choose a function $\psi$ and a constant $t_{\Omega}$ that satisfy (11.4). For example, for the unit ball we can take 
$\psi(x)=-x$ and $t_{\Omega}=1($ see $[21])$. Note that the set

$$
\bigcup_{0<t<t_{\Omega}}\{x+t(\psi(x)+B)\}
$$

is the union of a cone of altitude $|\psi(x)|$ and the ball of radius $t_{\Omega}$ centered at $x+t_{\Omega} \psi(x)$ if $|\psi(x)|>1$, and the ball of radius $t_{\Omega}$ centered at $x+t_{\Omega} \psi(x)$ if $|\psi(x)| \leq 1$.

Let $\varphi$ and $K$ be the functions defined in Lemma 2.3. Then Muramatu's integral formula for $f \in W_{p}^{m}(\Omega)$ takes a slightly more complicated form:

$$
\begin{aligned}
f= & \int_{0}^{\lambda} \sum_{|\beta|=m} t^{m}\left[\int_{\Omega} t^{-d} K_{\beta}\left(\frac{x-y}{t}+\psi(x)\right) \partial^{\beta} f(y) d y\right] \frac{d t}{t} \\
& +\int_{\Omega} \lambda^{-d} \varphi\left(\frac{x-y}{t}+\psi(x)\right) f(y) d y
\end{aligned}
$$

or more generally

$$
\begin{aligned}
\partial^{\alpha} f= & \int_{0}^{\lambda} \sum_{|\beta|=m} t^{m-|\alpha|}\left[\int_{\Omega} t^{-d} K_{\beta}^{(\alpha)}\left(\frac{x-y}{t}+\psi(x)\right) \partial^{\beta} f(y) d y\right] \frac{d t}{t} \\
& +\lambda^{-|\alpha|} \int_{\Omega} \lambda^{-d} \varphi^{(\alpha)}\left(\frac{x-y}{t}+\psi(x)\right) f(y) d y
\end{aligned}
$$

for $|\alpha| \leq m$ and $0<\lambda \leq t_{\Omega}$. We can prove (11.6) in the same way as Theorem 2.5, since the argument involves derivatives only with respect to $t$ and $y$.

For bounded domains we must note that the range of $\lambda$ is confined to a bounded interval. For this reason we obtain some results in weaker forms. For example, the interpolation inequality (2.18) holds if we replace $\left\|\nabla^{m} f\right\|_{L_{p}(\Omega)}$ by $\|f\|_{W_{p}^{m}(\Omega)}$.

The appearance of $\psi(x)$ causes no trouble, although it makes calculations longer. When $x$-derivatives are involved, we have extra terms which come from the drivatives of $\psi$, as observed in the calculation

$$
\begin{aligned}
& \partial_{x_{j}}\left\{K_{\beta}(\Psi(t, x, y))\right\} \\
& =t^{-1}\left(\partial_{j} K_{\beta}\right)(\Psi(t, x, y))+\sum_{i=1}^{d}\left(\partial_{i} K_{\beta}\right)(\Psi(t, x, y)) \partial_{j} \psi_{i}(x) \\
& =: J_{1}+J_{2},
\end{aligned}
$$

where $\Psi(t, x, y)=(x-y) / t+\psi(x)$ and $\psi(x)=\left(\psi_{1}(x), \ldots, \psi_{d}(x)\right)$. However, $J_{2}$, which consists of extra terms, can be handled easily, since $J_{2}$ does not contain the factor $t^{-1}$ unlike $J_{1}$, and hence $J_{2}$ is more $t$-integrable over $(0, \lambda]$ than $J_{1}$.

When $x$-derivatives are not involved, we can argue in the same way as in the case of special Lipschitz domain. To see this, we give a proof that $f \in W_{p}^{m}(\Omega)$ satisfies

$$
|\nabla f(x)| \leq C\left(\lambda^{-1-d / p}\|f\|_{L_{p}(\Omega)}+\lambda^{m-1-d / p}\left\|\nabla^{m} f\right\|_{L_{p}(\Omega)}\right)
$$

for $x \in \Omega, 0<\lambda \leq t_{\Omega}$ if $m-d / p>1$. 
To evaluate the first term on the right-hand side of (11.6), we set

$$
\begin{aligned}
H(x, z) & =K_{\beta}^{(\alpha)}(x+\psi(z)), \\
F(x) & =\int_{0}^{\lambda} t^{m-|\alpha|}\left[\int_{\Omega} t^{-d} H\left(\frac{x-y}{t}, x\right) f^{(\beta)}(y) d y\right] \frac{d t}{t}
\end{aligned}
$$

for $|\alpha|=1$ and $|\beta|=m$. Taking into account that $H((x-y) / t, x) \neq 0$ implies

$$
(x-y) / t \in-\psi(x)+B \subset\left(1+\|\psi\|_{L_{\infty}}\right) B,
$$

we write $\chi$ for the characteristic function of the ball $\left(1+\|\psi\|_{L_{\infty}}\right) B$. Then

$$
\begin{aligned}
|F(x)| & \leq\|H\|_{L_{\infty}} \int_{0}^{\lambda} t^{m-|\alpha|}\left[\int_{\Omega} t^{-d} \chi\left(\frac{x-y}{t}\right)\left|f^{(\beta)}(y)\right| d y\right] \frac{d t}{t} . \\
& \leq\|H\|_{L_{\infty}}\|\chi\|_{L_{p^{\prime}}}\left\|f^{(\beta)}\right\|_{L_{p}} \int_{0}^{\lambda} t^{m-1-d / p} \frac{d t}{t} \\
& \leq C \lambda^{m-1-d / p}\left\|f^{(\beta)}\right\|_{L_{p}}
\end{aligned}
$$

with $1 / p+1 / p^{\prime}=1$. Evaluating the second term on the right-hand side of (11.6) in a similar way, we obtain (11.7).

\section{Appendix}

We will complete the proof of Theorem 6.1 by deriving inequality (6.3) for the case $p \neq 2$. To this end we cite the theorem on page 29 in [30].

Theorem 12.1. Let $K \in \mathcal{S}\left(\mathbb{R}^{d}\right)$, the Schwartz space of rapidly decreasing functions, and define $T$ by

$$
T f(x)=K * f(x), \quad \text { for } f \in L_{p}(\Omega)
$$

Set

$$
M_{1}=\sup _{x \in \mathbb{R}^{d}}|x|^{d+1}|\nabla K(x)|, \quad M_{2}=\|\mathcal{F} K\|_{L_{\infty}\left(\mathbb{R}^{d}\right)} .
$$

Then for all $p \in(1, \infty)$ there exists $C=C\left(p, d, M_{1}, M_{2}\right)$ such that

$$
\|T f\|_{L_{p}\left(\mathbb{R}^{d}\right)} \leq C\|f\|_{L_{p}\left(\mathbb{R}^{d}\right)}, \quad \text { for } f \in L_{p}\left(\mathbb{R}^{d}\right) .
$$

Proof of inequality (6.3). We set $\tilde{K}(x)=\int_{\epsilon}^{\lambda} t^{z} K_{t}(x) t^{-1} d t$ for $0<\epsilon<\lambda$. By Theorem 12.1 we need only show that the two constants

$$
M_{1}=\sup _{x \in \mathbb{R}^{d}}|x|^{d+1}|\nabla \tilde{K}(x)|, \quad M_{2}=\|\mathcal{F} \tilde{K}\|_{L_{\infty}}
$$

are bounded by a constant depending only on $d, \lambda^{b}$ and $K$. We have already done so 
for $M_{2}$ in the proof of Theorem 6.1. As for $M_{1}$, we use the inequality $|x|^{d}\left|\partial_{j} K(x)\right| \leq$ $C\left(1+|x|^{2}\right)^{-1}$ with $C$ depending only on $K$ and $d$, which follows from $K \in \mathcal{S}\left(\mathbb{R}^{d}\right)$. Then

$$
\begin{aligned}
|x|^{d+1}\left|\partial_{j} \tilde{K}(x)\right| & =\left|\int_{\epsilon}^{\lambda} t^{z}\left(\frac{|x|}{t}\right)^{d+1} \partial_{j} K\left(\frac{x}{t}\right) \frac{d t}{t}\right| \\
& \leq C \int_{\epsilon}^{\lambda} t^{\operatorname{Re} z} \frac{|x| / t}{1+(|x| / t)^{2}} \frac{d t}{t} \\
& \leq C \max \left\{\lambda^{b}, 1\right\} \int_{0}^{\infty} \frac{d s}{1+s^{2}}
\end{aligned}
$$

which gives the desired estimate.

\section{Acknowledgments}

This research was supported by JSPS Grant-in-Aid for Scientific Research (C) 23540225. I am very grateful to Professor Shizuo Miyajima for inviting me to give an intensive course on Sobolev spaces at Tokyo University of Science in 2013. This paper extends and improves the lecture notes to a large extent. I appreciate the valuable comments and suggestions made by the referee.

\section{References}

[1] R.A. Adams, J.F. Fournier, Sobolev Spaces. 2nd Edition, Academic Press, Amsterdam, 2003.

[2] S. Agmon, Lectures on Elliptic Boundary Value Problems. AMS, Providence, RI, 2010.

[3] J. Bergh, J. Löfström, Interpolation Spaces - An Introduction. Springer, Berlin/New York, 1976.

[4] O.V. Besov, V.P. Il'in, S.M. Nikol'skii, Integral Representations of Functions and Imbedding Theorems, Vol. I. translated from the Russian, John Wiley \& Sons, New York, 1978.

[5] O.V. Besov, V.P. Il'in, S.M. Nikol'skii, Integral Representations of Functions and Imbedding Theorems, Vol. II. translated from the Russian, John Wiley \& Sons, New York, 1979.

[6] H. Brezis, Functional Analysis, Sobolev Spaces and Partial Differential Equations. Springer, New York, 2011.

[7] V.I. Burenkov, Sobolev Spaces on Domains. Teubner, Stuttgart, 1998.

[8] E. Di Nezza, G. Palatucci, E. Valdinoci, Hitchhiker's guide to the fractional Sobolev spaces. Bull. Sci. Math. 136 (2012), 521-573.

[9] L.C. Evans, Partial Differential Equations. 2nd Edition, AMS, Providence, RI, 2010.

[10] D.E. Edmunds, W.D. Evans, Spectral Theory and Differential Operators. Oxford University Press, Oxford, 1987.

[11] G.B. Folland, Introduction to Partial Differential Equations. 2nd Edition, Princeton University Press, Princeton, 1995. 
[12] G.B. Folland, Real Analysis. Modern Techniques and their Applications. 2nd Edition, Wiley, New York, 1999.

[13] A. Friedman, Partial Differential Equations. Holt, Rinehart and Winston, New York, 1969.

[14] D. Gilbarg, N.S. Trudinger, Elliptic Partial Differential Equations of Second Order. reprint of the 1998 edition, Springer, Berlin, 2001.

[15] D.D. Haroske, H. Triebel, Distributions, Sobolev Spaces, Elliptic Equations. EMS, Zürich, 2008.

[16] N.V. Krylov, Lectures on Elliptic and Parabolic Equations in Sobolev Spaces. AMS, Providence, RI, 2008.

[17] G. Leoni, A First Course in Sobolev Spaces. AMS, Providence, RI, 2009.

[18] V. Maz'ya, Sobolev Spaces with Applications to Elliptic Partial Differential Equations. Springer, New York, 2011.

[19] W. McLean, Strongly Elliptic Systems and Boundary Integral Equations. Cambridge University Press, Cambridge, 2000.

[20] Y. Miyazaki, New proofs of the trace theorem of Sobolev spaces. Proc. Japan Acad. 84 (2008), 112-116.

[21] Y. Miyazaki, Sobolev trace theorem and the Dirichlet problem in a ball. Int. J. Math. Anal. 10 (2016), 1173-1188.

[22] T. Muramatu, On Besov spaces and Sobolev spaces of generalized functions defined on a general region. Publ. Res. Inst. Math. Sci. 9 (1974), 325-396.

[23] T. Muramatu, On Sobolev spaces and Besov spaces (in Japanese). Sūgaku 27 (1975), $142-157$.

[24] T. Muramatu, Partial differential equations (2). in: K. Yosida, S. Ito (Ed.), Kansu Kaiseki to Bibun Hoteisiki (in Japanese) [Functional Analysis and Differential Equations], Iwanami Shoten, Tokyo, 1976, pp.267-330.

[25] T. Muramatu, Hokan Kukanron to Senkei Sayoso (in Japanese) [Theory of Interpolation Spaces and Linear Operators]. Kinokuniya, Tokyo, 1985.

[26] T. Runst, W. Sickel, Sobolev Spaces of Fractional Order, Nemytskij Operators, and Nonlinear Partial Differential Equations. Walter de Gruyter \& Co., Berlin, 1996.

[27] V.S. Rychkov, On restrictions and extensions of the Besov and Triebel-Lizorkin spaces with respect to Lipschitz domains. J. London Math. Soc. 60 (1999), 237-257.

[28] L. Saloff-Coste, Aspects of Sobolev-type Inequalities. Cambridge University Press, Cambridge, 2002.

[29] E.M. Stein, The characterization of functions arising as potentials II. Bull. Amer. Math. Soc. 68 (1962), 577-582.

[30] E.M. Stein, Singular Integrals and Differentiability Properties of Functions. Princeton University Press, Princeton, 1970.

[31] H. Tanabe, Functional Analytic Methods for Partial Differential Equations. Marcel Dekker, New York, 1997.

[32] L. Tartar, An Introduction to Sobolev Spaces and Interpolation Spaces. Springer, Berlin/New York, 2007. 
[33] M.E. Taylor, Partial Differential Equations I, Basic theory. 2nd Edition, Springer, New York, 2011.

[34] M.E. Taylor, Partial Differential Equations III, Nonlinear equations. 2nd Edition, Springer, New York, 2011.

[35] H. Triebel, Interpolation Theory, Function Spaces, Differential Operators. NorthHolland, Amsterdam, 1978.

[36] H. Triebel, Theory of Function Spaces. Birkhäuser, Basel, 1983.

[37] H. Triebel, Theory of Function Spaces II. Birkhäuser, Basel, 1992.

[38] H. Triebel, The Structure of Functions. Birkhäuser, Basel, 2001.

[39] H. Triebel, Theory of Function Spaces III. Birkhäuser, Basel, 2006.

[40] W.P. Ziemer, Weakly Differentiable Functions. Sobolev Spaces and Functions of Bounded Variation. Springer, New York, 1989.

Yoichi Miyazaki

School of Dentistry

Nihon University

1-8-13 Kanda-Surugadai

Chiyoda-ku, Tokyo 101-8310

Japan

e-mail: miyazaki.yoichi86@nihon-u.ac.jp

Received: December 11, 2016.

Accepted: April 26, 2017.

Open Access This article is distributed under the terms of the Creative Commons Attribution 4.0 International License (http://creativecommons.org/licenses/by/4.0/), which permits unrestricted use, distribution, and reproduction in any medium, provided you give appropriate credit to the original author(s) and the source, provide a link to the Creative Commons license, and indicate if changes were made. 